TRANSACTIONS OF THE

AMERICAN MATHEMATICAL SOCIETY

Volume 348, Number 6, June 1996

\title{
SPECIAL VALUES OF SYMMETRIC HYPERGEOMETRIC FUNCTIONS
}

\author{
FRANCESCO BALDASSARRI
}

Dedicated to Professor Bernard Dwork on his 73rd birthday

\begin{abstract}
We discuss the $p$-adic formula (0.3) of P. Th. Young, in the framework of Dwork's theory of the hypergeometric equation. We show that it gives the value at 0 of the Frobenius automorphism of the unit root subcrystal of the hypergeometric crystal. The unit disk at 0 is in fact singular for the differential equation under consideration, so that it's not a priori clear that the Frobenius structure should extend to that disk. But the singularity is logarith$m i c$, and it extends to a divisor with normal crossings relative to $\mathbf{Z}_{p}$ in $\mathbf{P}_{\mathbf{Z}_{p}}^{1}$. We show that whenever the unit root subcrystal of the hypergeometric system has generically rank 1, it actually extends as a logarithmic F-subcrystal to the unit disk at 0 . So, in these optics, "singular classes are not supersingular". If, in particular, the holomorphic solution at 0 is bounded, the extended logarithmic $F$-crystal has no singualrity in the residue class of 0 , so that it is an $F$-crystal in the usual sense and the Frobenius operation is holomorphic. We examine in detail its analytic form.
\end{abstract}

\section{INTRODUCTION}

In a recent article P. Th. Young, on the line of previous work by N. Koblitz $[\mathrm{Ko}]$ and J. Diamond [D], used some combinatorial identities and a principle of $p$-adic continuity in all variables to compute special values of a certain function $\mathcal{F}(a, b, c ; \lambda)$ related to the classical Gauss hypergeometric function $F(a, b, c ; \lambda)$. As in $\left[p\right.$-DE IV], the function $\mathcal{F}(a, b, c ; \lambda)$, for $a, b, c \in \mathbf{Z}_{p}, c \notin \mathbf{Z}_{\leq 0}$, is defined to be the maximal $p$-adic analytic extension of the ratio

$$
\frac{F(a, b, c ; \lambda)}{F\left(a^{\prime}, b^{\prime}, c^{\prime} ; \lambda^{p}\right)},
$$

where for $a \in \mathbf{Z}_{p}, a^{\prime} \in \mathbf{Z}_{p}$ is uniquely defined by the condition that

$$
p a^{\prime}-a=\mu_{a} \in\{0,1, \ldots, p-1\} .
$$

(We also recursively define $a^{(0)}=a$, and $a^{(i+1)}=\left(a^{(i)}\right)^{\prime}$, for $i=0,1, \ldots$ ) In particular, Young obtained the formula (see [Y1, Th. 3.1] and (5.3.1) below)

$$
\mathcal{F}\left(\frac{2 \alpha}{p-1}, \frac{\beta}{p-1}, 1+\frac{2 \alpha-\beta}{p-1} ;-1\right)=(-1)^{\alpha} \frac{\Gamma_{p}\left(\frac{\alpha}{p-1}\right) \Gamma_{p}\left(\frac{\beta-\alpha}{p-1}\right)}{\Gamma_{p}\left(\frac{2 \alpha}{p-1}\right) \Gamma_{p}\left(\frac{\beta-2 \alpha}{p-1}\right)}
$$

Received by the editors November 15, 1994.

1991 Mathematics Subject Classification. Primary 11T23, 11S31, 12H25, 14F30.

(C)1996 American Mathematical Society 
(where $\Gamma_{p}$ denotes the Morita $p$-adic gamma function), for any $\alpha, \beta \in \mathbf{Z}$ such that

$$
0<2 \alpha \leq \beta<p-1 \text { and } 2(\beta-\alpha)<p-1 .
$$

(Actually, the previous assumptions could be relaxed to be

$$
0 \leq 2 \alpha \leq \beta<p-1 \text { and } 2(\beta-\alpha) \leq p-1
$$

( $c f$. Theorem 5.3), for $p \geq 7$ ). When $(a, b, c)$ are rational, the differential equation satisfied by $F(a, b, c ; \lambda)$ was known, since the time of Euler, to express the variation w.r.t. to the parameter $\lambda$ of two cohomology classes on a variable algebraic curve $y=f_{a, b, c}(x, \lambda)$. For more general $(a, b, c)$, the analogues of the previous classes live in a twisted cohomology space for the $x$-projective line with a ramification point varying with $\lambda$, the cohomology being taken relative to the $\lambda$-line. The present paper originated from our attempt to gain a full cohomological understanding of the results of [Y1] and [Y2]. We work, naturally, in the framework of Dwork's theory of generalized hypergeometric equations ([GHF], $[\mathrm{LDE}]$ ), which encompasses both the classical algebraic and the $p$-adic cohomological theories. The main points to clarify were:

1. the relevance of the Frobenius structure of the hypergeometric system to a statement like (0.3);

2. the relevance of the "admissible" factorization of a linear differential operator according to the growth of solutions ([DR], [Ch, Prop. 4.2.1]) to the same statement;

3. more generally, the question of uniformity of the previous factorization when applied, say, to the family of Gauss hypergeometric operators, with respect to the variables $(a, b, c)$;

4. the notion of supersingularity of a singular fiber [Dw2];

5. the possibility, in certain cases, of explicitly computing the eigenvalues of Frobenius acting on a special fiber.

In particular, point 1 should clarify the role played in the proof of (0.3) by the Boyarsky principle, asserting the continuous dependence of the Frobenius matrix upon $(a, b, c ; \lambda)$ [GHF, Introduction and Th. 4.7.1].

Point 2 plays an important role here in that the main condition under which we work is

$$
\mu_{c^{(i)}}>\max \left(\mu_{a^{(i)}}, \mu_{b^{(i)}}\right)
$$

for any $i=0,1, \ldots$. This condition guarantees that the holomorphic solution of the hypergeometric operator at 0 (that is, $F(a, b, c ; \lambda)$ ) is a bounded holomorphic function on $D\left(0,1^{-}\right)=\left\{\lambda \in \mathbf{C}_{p}|| \lambda \mid<1\right\}$ [ $p$-DE IV, Lemma 2.2]. When $a, b, c \in$ $\frac{1}{p-1} \mathbf{Z}$ condition (0.5) (in this case independent of $i$ ) guarantees that the (rank 1) unit root $F$-subcrystal of the hypergeometric $F$-crystal extends to the singular class $D\left(0,1^{-}\right)$. We will come back to this point in $[\mathrm{Ba}]$, where the more general notion of logarithmic (realization of an overconvergent) $F$-(iso)crystal is introduced [Sch], and where $(0.5)$ is seen as the condition for the unit root logarithmic $F$-subcrystal of the hypergeometric logarithmic $F$-crystal to actually be an $F$-crystal in the usual sense in a $p$-adic formal neighborhood of 0 .

In the present article, we will not be concerned with point 4 above. The reason is that that problem does not appear for the (singular) class of 0 unless one deals 
with an iterate of Frobenius, $\lambda \longmapsto \lambda^{q}, q=p^{f}, f \in \mathbf{Z}_{>1}$. This might be the best approach ( $c f$. [LDE, Chap. 9], [Dw2]) when $a, b, c$ are in $\frac{1}{q-1} \mathbf{Z} \cap(0,1)$. In that case one would consider

$$
\mathcal{F}^{(f)}(a, b, c ; \lambda)=\frac{F(a, b, c ; \lambda)}{F\left(a^{(f)}, b^{(f)}, c^{(f)} ; \lambda^{q}\right)}
$$

The condition for $\mathcal{F}^{(f)}(a, b, c ; \lambda)$ to extend to an admissible domain of analyticity would then consist of two separate assumptions. The first is $(0.5)$ for $i=f-1$, which already guarantees 1-dimensionality of the space of bounded solutions of the system at a generic point. A second condition is needed to guarantee nonsupersingularity of the class of 0 , that is, the existence of a bounded solution of the system in $D\left(0,1^{-}\right)$. The condition says that the order of zero at $\lambda=0$ of the lowerright entry of the Frobenius matrix shouldn't exceed $c(q-1)$ [Dw2, Def. 1.11]. This is certainly the case if we insist that condition $(0.5)$ holds for all $i=0, \ldots, f-1$. This is the viewpoint of this article and of [p-DE IV]. A separate question is the one of non-supersingularity of the non-singular point $\lambda_{0}$ at which we want to evaluate $\mathcal{F}(a, b, c ; \lambda)$. In the case considered here, $\lambda_{0}=-1$ and $c \pm(a-b) \in \mathbf{Z}$, this is automatic (cf. (2.31) and (2.36)). In the case considered by [Ko] and [D], $\lambda_{0}=1$ is singular, so condition (0.5) appears combined with a similar condition [D, (iii) of Thm. 1.1] at 1, to guarantee that the above mentioned unit root $F$-crystal also extends to a $p$-adic formal neighbourhood of 1 . Again, non-supersingularity of the class of 1 follows from that condition.

While problem 5 also becomes more interesting in the case of a singular fiber, where Frobenius operates on the "eigenvectors of local monodromy" ( $c f$. [GHF, Lemmas 24.3 and 24.5.8]) in this paper, motivated by formula (0.3), we only discuss the non-singular fiber at $\lambda_{0}=-1$. We carefully analyze the relation between the value

$$
\mathcal{F}\left(\frac{2 \alpha}{p-1}, \frac{\beta}{p-1}, 1+\frac{2 \alpha-\beta}{p-1} ;-1\right)=\mathcal{F}\left(\frac{\beta}{p-1}, \frac{2 \alpha}{p-1}, 1+\frac{2 \alpha-\beta}{p-1} ;-1\right)
$$

and the unit root of the $L$-function

$$
L(t)=\exp \sum_{s=1}^{\infty} S_{\alpha, \beta, s} \frac{t^{s}}{s},
$$

where

$$
S_{\alpha, \beta, s}=\sum_{x \in \mathbf{F}_{p^{s}} \backslash\{0,1,-1\}} \omega^{-2 \alpha}\left(\operatorname{Tr}_{\mathbf{F}_{p^{s}} / \mathbf{F}_{p}}(x)\right) \omega^{-\beta}\left(\operatorname{Tr}_{\mathbf{F}_{p^{s}} / \mathbf{F}_{p}}\left(1-x^{2}\right)\right)
$$

and $\omega: \mathbf{F}_{p}^{\times} \longrightarrow \mathbf{Q}\left(\zeta_{p-1}\right)^{\times}$denotes the Teichmüller character. An elementary calculation, taking into account the classical relation between Gauss and Jacobi sums, the Hasse-Davenport relation and the Gross-Koblitz formula [GrKo], gives

$$
L(t)=\left(1-\sigma_{1}(\alpha, \beta) t\right)\left(1-\sigma_{2}(\alpha, \beta) t\right)
$$


with

$$
\sigma_{1}(\alpha, \beta)=(-1)^{\alpha} \frac{\Gamma_{p}\left(\frac{\alpha}{p-1}\right) \Gamma_{p}\left(\frac{\beta-\alpha}{p-1}\right)}{\Gamma_{p}\left(\frac{\beta}{p-1}\right)},
$$

$$
\sigma_{2}(\alpha, \beta)=-p(-1)^{\alpha+\frac{p-1}{2}} \frac{\Gamma_{p}\left(\frac{\alpha+\frac{p-1}{2}}{p-1}\right) \Gamma_{p}\left(\frac{\beta-\alpha+\frac{p-1}{2}}{p-1}\right)}{\Gamma_{p}\left(\frac{\beta}{p-1}\right)} .
$$

In the notation of $(2.37 .1,2)$

$$
\begin{aligned}
\mathbf{B}\left(\frac{\beta}{p-1}, \frac{2 \alpha}{p-1}, 1\right. & \left.+\frac{2 \alpha-\beta}{p-1} ; \frac{\beta}{p-1}, \frac{2 \alpha}{p-1}, 1+\frac{2 \alpha-\beta}{p-1} ;-1\right) \\
& =\left(\begin{array}{cc}
\sigma_{2}(\alpha, \beta) & 0 \\
* & \sigma_{1}(\alpha, \beta),
\end{array}\right)
\end{aligned}
$$

so that

$$
L(t)=\operatorname{det}\left(I_{2}-t \mathbf{B}\left(\frac{\beta}{p-1}, \frac{2 \alpha}{p-1}, 1+\frac{2 \alpha-\beta}{p-1} ; \frac{\beta}{p-1}, \frac{2 \alpha}{p-1}, 1+\frac{2 \alpha-\beta}{p-1} ;-1\right)\right) .
$$

The fact that the evaluation of $\mathcal{F}(a, b, c ; \lambda)$ takes place at $\lambda=-1$, which is not a singular point of the differential equation satisfied by $F(a, b, c ; \lambda)$, reduces the calculation of the eigenvalues of Frobenius operating on local solutions of the differential equation to a problem of character sums on the special fiber, which, in our particular case, is elementary. At a singular fiber $\lambda_{0}$, the eigenvalues of Frobenius operating on the eigenfunctions of local monodromy have only been determined when $\lambda_{0}=0$ ([LDE, Chaps. 25-26]), via their interpretation on the cohomology of the special fiber. The calculation when $\lambda_{0}=1$ or $\infty$ may then be performed with the help of the theorem in $[\mathrm{Ku}, \S 4]$. We plan to give full details in [Ba].

The speciality of the fiber of the relative cohomology bundle at $\lambda=-1$ in the case under consideration, namely $c \pm(a-b) \in \mathbf{Z}$, lies in the fact that it is "symmetric", by which we mean that it is equipped with an involution $\tau$ (see [Y1,3.15] and diagram (1.5) below), commuting with the action of Frobenius. This involution splits the cohomology space at $\lambda=-1$ rationally in $(a, b, c)$ and offers the possibility of obtaining nice formulas separately for the two roots of Frobenius (see formulas (2.30) and (2.35) below). That these formulas should involve products of $p$-adic $\Gamma$ functions is then a priori clear because of the modular properties of the Frobenius matrix w.r.t. integral translation on $(a, b, c)$ ( $c f$. [GHF, Lemma 4.8.1] and [Sel]). These modular properties of the Frobenius matrix simply express the fact that Frobenius represents an intrinsic horizontal transformation of relative cohomology sheaves depending rationally upon $(a, b, c)$, whose matrix may be computed w.r.t. a lattice of natural bases. The same modular properties hold for the matrices representing the action of global monodromy on classical solutions, i.e. relating the eigenvectors of local monodromy at two distinct singular points [Po, Chap. VI, $\S 26]$, but their full potential does not seem to have been classically exploited.

We point out once again that the mere fact that the two eigenvalues of Frobenius at $\lambda=-1$ have $p$-adic absolute values 1 and $p^{-1}$, respectively, implies the existence 
of a rank 1 unit root $F$-subcrystal [Ka, $\S 4]$ of the hypergeometric system over an admissible open subset of the $\lambda$-line, containing the class of -1 . Under condition (0.5), the unit root $F$-crystal extends to the singular class of 0 . This is in the end the key ingredient that permits one to relate $\mathcal{F}(a, b, a-b+1 ;-1)$ to an entry of the Frobenius matrix at -1 .

The contribution of this paper to the previous problems is as follows. Section 1 analyzes the Frobenius matrix at the special symmetric fiber $\lambda=-1$, via the modular relations. This approach represents an alternative way to the direct elementary computation of the associated $L$-function. Section 2 reviews some of the results of Dwork contained, in two slightly different forms, in the two books [GHF] and [LDE]: we explicitly describe the dictionary between them. Section 3 represents an improvement on previous results of Dwork. Namely, we describe the Frobenius matrix uniformly for $(a, b, c ; \lambda)$ varying in a region of the form $\mathcal{H} \times S$, where $\mathcal{H}$ represents an affinoid domain in the $(a, b, c)$-space $\left(\mathbf{C}_{p}\right)^{3}$, and $S$ the complement in the rigid $\lambda$-line of the residue classes at 1 and at $\infty$. In particular, $S$ contains the singular residue class $D\left(0,1^{-}\right)$. This is new, since the theory of [LDE] and [Dw2] is not uniform in $(a, b, c)$, while the theory of [GHF] and [Dw1] does not analyze any full singular disk. Our main result is Corollary 3.37, a result which should at least be compared with the theorem in section 4 of [Dw1] and with Lemmas 24.4 and 24.5.8 of [LDE]. By the methods of [Ku] these results may be extended to the other singular classes as well. (In the class of 1 the correct Frobenius map to use on the $\lambda$-space should be close to $\lambda \longmapsto 1-(1-\lambda)^{p}$.) For this we refer to [Ba]. We also wish to point out that, at least when $p \geq 7$, our list of distinct cases is exhaustive, which also represents an improvement upon the classification of [LDE, Chap. 6]. In section 4 we examine the question of uniformity in $(a, b, c)$ of the growth decomposition. Here we examine hypergeometric equations in the so-called "split" cases, when at the generic point there are both bounded and unbounded solutions. We prove that the factorization according to the order of growth extends to the residue class of 0 . In section 5 , we finally show how to recover formula (0.3) from our discussion.

The study of the singular classes of 1 (and infinity), and its application yielding the formulas of Koblitz [Ko] and Diamond [D], is deferred to the previously mentioned article [Ba].

The author is grateful to Professor Dwork for his generous help in the revision of the original manuscript.

\section{List OF SYMBOLS}

$$
\mathbf{B}=\left(\begin{array}{ll}
\mathbf{B}_{1} & \mathbf{B}_{2} \\
\mathbf{B}_{3} & \mathbf{B}_{4}
\end{array}\right)
$$

$d(A, B)=$ distance between the points or subsets $A$ and $B$ of $\left(\mathbf{C}_{p}\right)^{n}$, any $n$;

$D=D\left(0,1^{-}\right)$

$D\left(0, \rho^{-}\right)=\left\{\lambda \in \mathbf{C}_{p}|| \lambda \mid<\rho\right\}$

$D_{\vec{a}, i}=x_{i} \partial / \partial x_{i}+\pi x_{i} \partial / \partial x_{i}(g(x, \lambda))+a_{i}$, for $i=1,2,3,4$,

Chap. 1 of $[\mathrm{GHF}]$

$D_{\vec{a}, i}^{*}$, for $i=1,2,3,4$,

$f=f(x, \lambda)$

(3.22.1) of $[\mathrm{GHF}]$

$\tilde{f}=\tilde{f}(x, \lambda)$

Chap. 1 of $[\mathrm{LDE}]$

$F(a, b, c ; \lambda)$

$F(x, \lambda)=\exp \pi\left(g(x, \lambda)-g\left(x^{p}, \lambda^{p}\right)\right)$

Prop. 4.2 .2 of $[\mathrm{GHF}]$ 
$g(x, \lambda)=x_{3}\left(x_{1}+x_{2}\right)+x_{4}\left(\lambda x_{1}+x_{2}\right)$

$\S 13.2$ of $[\mathrm{GHF}]$

$g(x)=g(x,-1)$

$G_{f, h}(x, \lambda)$

(3.7.1) of $[\mathrm{LDE}]$

$G_{\tilde{f}, \tilde{h}}(x, \lambda)$

$h=h(x, \lambda)$

$\tilde{h}=\tilde{h}(x, \lambda)$

$H=$ hyperplane $t_{1}+t_{2}=t_{3}+t_{4}$ in affine 4 -space

$H^{+}(\mathbf{Z})=H(\mathbf{Z}) \cap\left(\mathbf{Z}_{\geq 0}\right)^{4}$

$K=$ a finite extension of $\mathbf{Q}_{p}(\pi)$;

$k(\vec{u})=u_{3}+u_{4}$, for $u \in H(\mathbf{Z})$

$L$

$\operatorname{ord}(x)=-\log _{p}|x|$, for $x \in \mathbf{C}_{p}^{\times}$;

$p=$ an odd prime number;

$R=\mathbf{C}_{p}(\lambda)\left[x_{1} x_{3}, x_{1} x_{4}, x_{2} x_{3}, x_{2} x_{4}\right]$

$R^{\prime}=\mathbf{C}_{p}(\lambda)\left[x_{1} x_{3}, x_{1} x_{4}, x_{2} x_{3}, x_{2} x_{4},\left(x_{1} x_{2} x_{3} x_{4}\right)^{-1}\right]$

$R^{\prime *}$

$S$

$S_{\epsilon}, \epsilon \geq 0$,

$S^{\vec{\mu}}$

$S_{i}^{H}, i=1,2$,

$T_{I}$

$T_{I I}$

$U^{(0)}(a, b, c ; \lambda)$

Chap. 1 of $[\mathrm{GHF}]$

$u_{i}^{(0)}(a, b, c ; \lambda), i=1,2,3,4$,

$\mathcal{B}_{K}$

$\mathcal{B}_{K}^{(1)}$

$\mathcal{C}_{K}$

$\mathcal{C}_{K}^{(1)}$

[GHF, Chap. 1]

$\S 2.2$ of $[\mathrm{GHF}]$

(3.16) of [GHF]

$\mathcal{H}$

(3.14) and (3.27)

$\mathcal{H}_{\vec{\mu}}$

$\mathcal{H}^{\prime}$

$\mathcal{H}_{i}^{\prime}, i=1,2$,

$\mathcal{H}_{\vec{\nu}}^{\prime}$

$\mathcal{L}$

$\mathcal{L}_{\vec{\mu}}$

$\mathcal{K}_{\vec{a}, \lambda}^{\prime}=\bigcap_{i=1}^{4} \operatorname{Ker}_{R^{\prime *}} D_{\vec{a}, i}^{*}$

$\S 3.2$ of $[\mathrm{GHF}]$

$\mathcal{S}_{i}^{\prime}(-1), i=1,2$,

$\mathcal{S}$

Lemma 2.4

$\mathcal{S}^{\prime}$

$\mathcal{T}_{i}, i=1,2$,

$\mathcal{T}_{i}(-1), i=1,2$,

$\mathcal{W}_{\vec{a}}^{\prime}=\mathcal{W}_{\vec{a}, \lambda}^{\prime}=R^{\prime} / \sum_{i=1}^{4} D_{\vec{a}, i} R^{\prime}$

Lemma 2.5.2 and $\S 3.2$ of [GHF]

$\left(\mathbf{C}_{p},|-|\right)=$ completion of the algebraic closure of $\mathbf{Q}_{p},|p|=p^{-1}$;

$\mathbf{K}_{f}=\mathbf{K}_{f, \lambda}$

Chap. 2 and 4 of [LDE]

$\mathbf{L}=\mathbf{C}_{p}(\lambda)\left[x, \frac{1}{x(1-x)(1-\lambda x)}\right]$

Chap. 1 of $[\mathrm{LDE}]$

$\tilde{\mathbf{L}}=\mathbf{C}_{p}(\lambda)\left[x, \frac{1}{x(1+x)(1+\lambda x)}\right]$ 


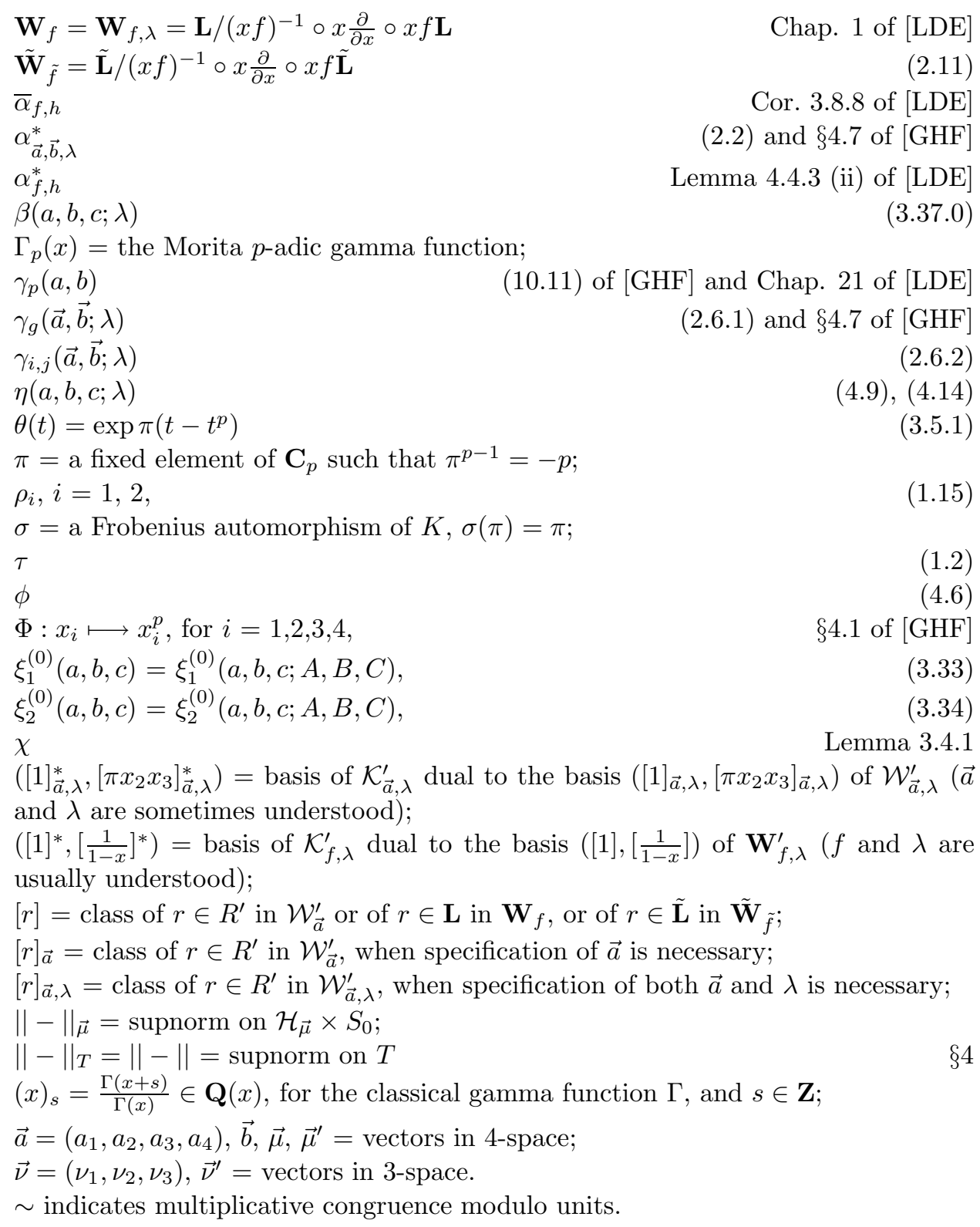

\section{Frobenius matrix on a Symmetric fiber OF EXPONENTIAL COHOMOLOGY SPACES}

We refer here to the computations of $\S 13.2$ of [GHF], with $a_{3}=a_{4}$ and $\lambda=-1$. We consider the linear $\mathbf{Z}$-subvariety $L$ of the affine 4 -space over $\mathbf{Z}$ defined by the equations:

$$
\begin{aligned}
t_{1}+t_{2} & =t_{3}+t_{4}, \\
t_{3} & =t_{4} .
\end{aligned}
$$


Notice that $L$ is a subvariety of the $\mathbf{Z}$-hyperplane $H$ of [GHF, Chap. 1]. We will consider pairs $(\vec{a}, \vec{b}) \in L\left(\mathbf{C}_{p}\right) \times L\left(\mathbf{C}_{p}\right)$, with $\vec{\mu}=p \vec{b}-\vec{a} \in \mathbf{Z}^{4}$. We consider the automorphism $\tau$ of $R^{\prime *}$, stable on $R^{\prime}$, such that

$$
\begin{array}{cc}
\tau\left(x_{1}\right)=-x_{1}, & \tau\left(x_{2}\right)=x_{2}, \\
\tau\left(x_{3}\right)=x_{4}, & \tau\left(x_{4}\right)=x_{3} .
\end{array}
$$

It's clear that $\tau: R^{\prime *} \longrightarrow R^{\prime *}$ is adjoint to $\tau: R^{\prime} \longrightarrow R^{\prime}$ in the natural pairing [GHF, 3.19] between $R^{\prime}$ and $R^{\prime *}$. Here $g=x_{3}\left(x_{1}+x_{2}\right)+x_{4}\left(-x_{1}+x_{2}\right)$, so that $g^{\tau}=g$. Then

$$
\begin{gathered}
\tau D_{\vec{a}, i} \tau=D_{\vec{a}, i}, \text { for } i=1,2, \\
\tau D_{\vec{a}, i}^{*} \tau=D_{\vec{a}, i}^{*}, \text { for } i=1,2, \\
\tau D_{\vec{a}, 3} \tau=D_{\vec{a}, 4}, \\
\tau D_{\vec{a}, 3}^{*} \tau=D_{\vec{a}, 4}^{*}, \\
\tau \Phi \tau=\Phi .
\end{gathered}
$$

Now

$$
\alpha_{\vec{a}, \vec{b},-1}^{*}: \mathcal{K}_{\vec{b},-1}^{\prime} \longrightarrow \mathcal{K}_{\vec{a},-1}^{\prime}
$$

is induced by $\delta_{g, \vec{\mu}}=x^{-\vec{\mu}} F(x, \lambda) \Phi$. But $\tau \delta_{g, \vec{\mu}} \tau=(-1)^{\mu_{1}} \delta_{g, \vec{\mu}}$. So, if $\mu_{1}$ is even we have commutative diagrams:

$$
\begin{array}{ccc}
\mathcal{K}_{\vec{b},-1}^{\prime} & \stackrel{\alpha_{\vec{a}, \vec{b},-1}^{*}}{\longrightarrow} & \mathcal{K}_{\vec{a},-1}^{\prime} \\
\tau \downarrow & & \downarrow \tau \\
\mathcal{K}_{\vec{b},-1}^{\prime} & \stackrel{\alpha_{\vec{a}, \vec{b},-1}^{*}}{\longrightarrow} & \mathcal{K}_{\vec{a},-1}^{\prime}
\end{array}
$$

We will therefore assume

$$
\mu_{1} \in 2 \mathbf{Z}
$$

(so in fact also $\mu_{2} \in 2 \mathbf{Z}$ ).

Since $\tau[1]_{\vec{a}}=[1]_{\vec{a}}$ and $\tau\left[\pi x_{2} x_{3}\right]_{\vec{a}}=-a_{2}[1]_{\vec{a}}-\left[\pi x_{2} x_{3}\right]_{\vec{a}}$, we see that $\tau\left[\pi x_{2} x_{3}\right]_{\vec{a}}^{*}=$ $-\left[\pi x_{2} x_{3}\right]_{\vec{a}}^{*}$. From this and the commutative diagram $(1.5)$, we see that $\gamma_{g}(\vec{a}, \vec{b} ;-1)$ is lower-triangular:

$$
\alpha_{\vec{a}, \vec{b},-1}^{*}\left([1]^{*},\left[\pi x_{2} x_{3}\right]^{*}\right)=\left([1]^{*},\left[\pi x_{2} x_{3}\right]^{*}\right) \gamma_{g}(\vec{a}, \vec{b} ;-1)
$$

(cf. $\S 4.7$ of $[\mathrm{GHF}])$.

Remark 1.7.1. The commutative diagram (1.5) shows that

$$
\left(\begin{array}{cc}
1 & 0 \\
-a_{2} & -1
\end{array}\right) \gamma_{g}(\vec{a}, \vec{b} ;-1)=\gamma_{g}(\vec{a}, \vec{b} ;-1)\left(\begin{array}{cc}
1 & 0 \\
-b_{2} & -1
\end{array}\right)
$$


and hence writing $\gamma_{g}=\left(\begin{array}{ll}\gamma_{11} & \gamma_{12} \\ \gamma_{21} & \gamma_{22}\end{array}\right)$, we find that $\gamma_{12}=0$ (as stated), but also $\gamma_{21}=\frac{1}{2}\left(\gamma_{22} b_{2}-\gamma_{11} a_{2}\right)$, i.e. $\gamma_{g}$ is determined by $\gamma_{11}$ and $\gamma_{22}$.

For $\vec{u} \in H(\mathbf{Z})$, we put

$$
k(\vec{u})=u_{3}+u_{4} .
$$

We examine the matrix of the mapping of multiplication by $\pi^{k(\vec{u})} x^{\vec{u}}$, for $\vec{u}=u^{\prime}, u^{\prime \prime}$, where $u^{\prime}=(2,0,1,1), u^{\prime \prime}=(0,2,1,1)$ :

$$
\begin{gathered}
\mathcal{W}_{\vec{a}+\vec{u}}^{\prime} \stackrel{\pi^{u_{3}+u_{4}} x^{\vec{u}}}{\longrightarrow} \mathcal{W}_{\vec{a}}^{\prime}, \\
\left(\begin{array}{c}
{[1]_{\vec{a}+\vec{u}}} \\
{\left[\pi x_{2} x_{3}\right]_{\vec{a}+\vec{u}}}
\end{array}\right) \longmapsto M(\vec{a}+\vec{u}, \vec{a} ;-1)\left(\begin{array}{c}
{[1]_{\vec{a}}} \\
{\left[\pi x_{2} x_{3}\right]_{\vec{a}}}
\end{array}\right),
\end{gathered}
$$

The computations in [GHF, loc. cit.] (after the harmless substitution $x_{i} \longmapsto \pi x_{i}$, for $i=3,4$ ) give

$$
\begin{aligned}
& M\left(\vec{a}+u^{\prime}, \vec{a} ;-1\right)=\left(\begin{array}{cc}
-\frac{a_{1} a_{3}}{2} & 0 \\
-\frac{a_{2} a_{3}}{4} & -a_{3} \frac{a_{1}+1}{2}
\end{array}\right), \\
& M\left(\vec{a}+u^{\prime \prime}, \vec{a} ;-1\right)=\left(\begin{array}{cc}
\frac{a_{2} a_{3}}{2} & 0 \\
-\frac{a_{2} a_{3}}{4} & a_{3} \frac{a_{2}+1}{2} .
\end{array}\right) .
\end{aligned}
$$

For the convenience of the reader, we review here how formulas (1.9) could be proven. We put $x_{i} x_{j}=x^{u^{(i, j)}}, M\left(\vec{a}+u^{(i, j)}, \vec{a} ;-1\right)=A_{i, j}(\vec{a})$, for $i=1,2, j=3,4$, and consider

$$
\mathcal{W}_{\vec{a}+u^{(i, j)}+u^{(h, k)}}^{\prime} \stackrel{\pi x_{h} x_{k}}{\longrightarrow} \mathcal{W}_{\vec{a}+u^{(i, j)}}^{\prime} \stackrel{\pi x_{i} x_{j}}{\longrightarrow} \mathcal{W}_{\vec{a}}^{\prime}
$$

More precisely:

$$
\mathcal{W}_{\vec{a}+u^{(i, j)}+u^{(h, k)}}^{\prime} \stackrel{\pi x_{h} x_{k}}{\longrightarrow} \mathcal{W}_{\vec{a}+u^{(i, j)}}^{\prime},
$$

$$
\begin{gathered}
\left(\begin{array}{c}
{[1]} \\
{\left[\pi x_{2} x_{3}\right]}
\end{array}\right) \longmapsto A_{h, k}\left(\vec{a}+u^{(i, j)}\right)\left(\begin{array}{c}
{[1]} \\
{\left[\pi x_{2} x_{3}\right]}
\end{array}\right), \\
\mathcal{W}_{\vec{a}+u^{(i, j)}}^{\prime} \stackrel{\pi x_{i} x_{j}}{\longrightarrow} \mathcal{W}_{\vec{a}}^{\prime},
\end{gathered}
$$

$$
\left(\begin{array}{c}
{[1]} \\
{\left[\pi x_{2} x_{3}\right]}
\end{array}\right) \longmapsto A_{i, j}(\vec{a})\left(\begin{array}{c}
{[1]} \\
{\left[\pi x_{2} x_{3}\right]}
\end{array}\right)
$$

so that

$$
\mathcal{W}_{\vec{a}+u^{(i, j)}+u^{(h, k)}}^{\prime} \stackrel{\pi^{2} x_{h} x_{k} x_{i} x_{j}}{\longrightarrow} \mathcal{W}_{\vec{a}}^{\prime}
$$

$$
\left(\begin{array}{c}
{[1]} \\
{\left[\pi x_{2} x_{3}\right]}
\end{array}\right) \longmapsto A_{h, k}\left(\vec{a}+u^{(i, j)}\right) A_{i, j}(\vec{a})\left(\begin{array}{c}
{[1]} \\
{\left[\pi x_{2} x_{3}\right]}
\end{array}\right)
$$


Then

$$
\begin{aligned}
& M\left(\vec{a}+u^{\prime}, \vec{a} ;-1\right)=A_{1,3}(\vec{a}+(1,0,0,1)) A_{1,4}(\vec{a}), \\
& M\left(\vec{a}+u^{\prime \prime}, \vec{a} ;-1\right)=A_{2,3}(\vec{a}+(0,1,0,1)) A_{2,4}(\vec{a}),
\end{aligned}
$$

and the formulas for $A_{i, j}(\vec{a})$ are explicitly given in [GHF, (13.2)] $\left(A_{i, j}(\vec{a})=z_{i j}\right.$, in the notation of loc. cit.), for $(i, j)=(2,3),(2,4),(1,4),(1,3)$. Of course, we must put $a_{3}=a_{4}$ and $\lambda=-1$ in those formulas.

So, in terms of the system of bases $\left(\begin{array}{c}{[1]_{\vec{a}}} \\ {\left[\pi x_{2} x_{3}\right]_{\vec{a}}}\end{array}\right)$ used in $[\mathrm{GHF}]$, we obtain for the matrices of multiplication by $\pi^{k(\vec{u})} x^{\vec{u}}$, for $\vec{u}=u^{\prime}, u^{\prime \prime}$, formulas (1.9).

Remark 1.10.6. The fact that $M(\vec{a}+\vec{u}, \vec{a} ;-1)$, for $\vec{u}$ in the $\mathbf{Z}$-span of $u^{\prime}$ and $u^{\prime \prime}$, is lower-triangular may be seen directly as in the previous remark 1.7.1. In fact, for such a $\vec{u}$, the map $\tau$ commutes with multiplication by $x^{\vec{u}}$. Hence writing

$$
M(\vec{a}+\vec{u}, \vec{a} ;-1)=\left(\begin{array}{ll}
M_{11} & M_{12} \\
M_{21} & M_{22}
\end{array}\right),
$$

we deduce that $M_{12}=0$ and $M_{21}=\frac{1}{2}\left(a_{2}\left(M_{22}-M_{11}\right)-M_{11} u_{2}\right)$. Once again $M(\vec{a}+\vec{u}, \vec{a} ;-1)$ is determined by $M_{11}$ and $M_{22}$.

We recall the determinant formula ( $\$ 13.6$ and Cor. 14.1.2 of $[\mathrm{GHF}]$ )

$$
\operatorname{det} \gamma_{g}(\vec{a}, \vec{b} ;-1)=p 2^{\mu_{1}+\mu_{2}}\left(2^{p-1}\right)^{-b_{1}-b_{2}} \prod_{i=1}^{4} \gamma_{p}\left(a_{i}, b_{i}\right) .
$$

The modular behaviour of $\gamma_{g}(\vec{a}, \vec{b} ;-1)$ w.r.t. integral translations in $\vec{a}$ is described by the formula ( $\$ 4.8$ of $[\mathrm{GHF}]$ )

$$
\gamma_{g}(\vec{a}+\vec{u}, \vec{b} ;-1)=M(\vec{a}+\vec{u}, \vec{a} ;-1) \gamma_{g}(\vec{a}, \vec{b} ;-1) \pi^{-k(\vec{u})} .
$$

It is apparent that these formulas are consistent with our previous calculations.

Formula (1.12) is of interest to us when $\vec{u}$ belongs to the $\mathbf{Z}$-span of $u^{\prime}, u^{\prime \prime}$. For such a $\vec{u}$, the formula involves lower-triangular matrices and spells out in term of the system of bases $\left([1]_{\vec{a}}^{*},\left[\pi x_{2} x_{3}\right]_{\vec{a}}^{*}\right)$ the commutative diagram

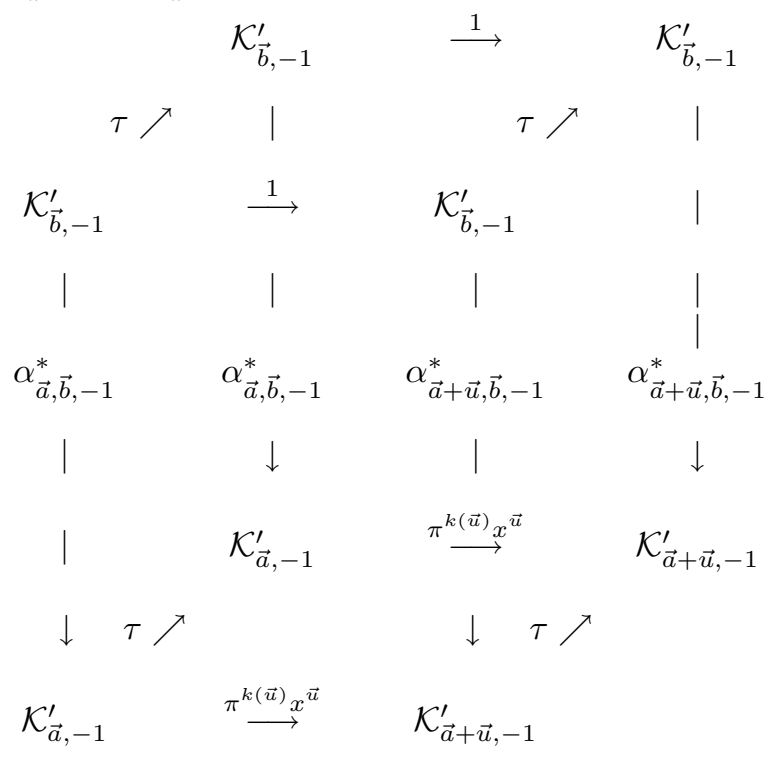


We put $\rho=p^{-\frac{1}{p-1}-\frac{1}{p}}$ and define for $\vec{\mu} \in \mathbf{Z}^{4} \cap L$ with $\mu_{1} \in 2 \mathbf{Z}$ :

$$
\mathcal{L}_{\vec{\mu}}=\left\{(a, b) \in L\left(\mathbf{C}_{p}\right) \times L\left(\mathbf{C}_{p}\right) \mid p \vec{b}-\vec{a}=\vec{\mu}, d(\vec{a},-\vec{\mu})<\rho\right\},
$$

$$
\mathcal{L}=\bigcup_{\vec{\mu} \in L(\mathbf{Z}), \mu_{1} \in 2 \mathbf{Z}} \mathcal{L}_{\vec{\mu}}
$$

We define functions on $\mathcal{L}$ by

$$
\begin{aligned}
& \rho_{1}(\vec{a}, \vec{b})=(-1)^{\frac{\mu_{1}}{2}} \gamma_{p}\left(\frac{a_{1}}{2}, \frac{b_{1}}{2}\right) \gamma_{p}\left(\frac{a_{2}}{2}, \frac{b_{2}}{2}\right) \gamma_{p}\left(a_{3}, b_{3}\right), \\
& \rho_{2}(\vec{a}, \vec{b})=(-1)^{\frac{\mu_{1}+p-1}{2}} \gamma_{p}\left(\frac{a_{1}+1}{2}, \frac{b_{1}+1}{2}\right) \gamma_{p}\left(\frac{a_{2}+1}{2}, \frac{b_{2}+1}{2}\right) \gamma_{p}\left(a_{3}, b_{3}\right) .
\end{aligned}
$$

Then:

$$
\rho_{1}(0,0)=1, \quad \rho_{2}(0,0)=(-1)^{\frac{p-1}{2}} \gamma_{p}\left(\frac{1}{2}, \frac{1}{2}\right)^{2}=p .
$$

Since ( $\S 10.12$ of $[\mathrm{GHF}])$, for $s, t \in \mathbf{Z}$,

$$
\gamma_{p}\left(b+s, b^{\prime}+t\right)=\gamma_{p}\left(b, b^{\prime}\right) \frac{(b)_{s}}{(-\pi)^{s}} \frac{(-\pi)^{t}}{\left(b^{\prime}\right)_{t}},
$$

we have:

$$
\begin{aligned}
\rho_{1}\left(\vec{a}+u^{\prime}, \vec{b}\right) & =-\frac{a_{1} a_{3}}{2 \pi^{2}} \rho_{1}(\vec{a}, \vec{b}), \\
\rho_{1}\left(\vec{a}+u^{\prime \prime}, \vec{b}\right) & =-\frac{a_{2} a_{3}}{2 \pi^{2}} \rho_{1}(\vec{a}, \vec{b}), \\
\rho_{2}\left(\vec{a}+u^{\prime}, \vec{b}\right) & =-\frac{\left(a_{1}+1\right) a_{3}}{2 \pi^{2}} \rho_{2}(\vec{a}, \vec{b}), \\
\rho_{2}\left(\vec{a}+u^{\prime \prime}, \vec{b}\right) & =-\frac{\left(a_{2}+1\right) a_{3}}{2 \pi^{2}} \rho_{2}(\vec{a}, \vec{b}) .
\end{aligned}
$$

Therefore $\rho_{1}, \rho_{2}$ are precisely the diagonal terms of $\gamma_{g}(\vec{a}, \vec{b} ;-1)$ :

$$
\gamma_{g}(\vec{a}, \vec{b} ;-1)=\left(\begin{array}{cc}
\rho_{1}(\vec{a}, \vec{b}) & 0 \\
* & \rho_{2}(\vec{a}, \vec{b})
\end{array}\right) .
$$

For fixed $\mu \in \mathbf{Z}$, the function $\gamma_{p}(b, c)$ with $p c-b=\mu$ is a meromorphic function of $b \in \mathbf{C}_{p}$ in the disk $|b+\mu|<\rho$. We recall for future use from [GHF, 10.7] that

$$
\begin{aligned}
\gamma_{p}(b, c) \gamma_{p}\left(b+\frac{1}{2}, c+\frac{1}{2}\right) & =\gamma_{p}(2 b, 2 c) 4^{\mu}\left(4^{1-p}\right)^{c} \gamma_{p}\left(\frac{1}{2}, \frac{1}{2}\right), \\
\gamma_{p}(b, c) \gamma_{p}(1-b, 1-c) & =p(-1)^{\mu} \\
\gamma_{p}\left(\frac{1}{2}, \frac{1}{2}\right)^{2} & =(-1)^{\frac{p-1}{2}} p
\end{aligned}
$$


for $b, c \in \mathbf{C}_{p}, p c-b=\mu \in \mathbf{Z},|b+\mu|<\rho$. In the same notation

$$
\pi^{-\mu} \gamma_{p}(b, c) \sim \begin{cases}1 & \text { if } \mu \in[0, p-1], \\ \frac{1}{p(c-1)} & \text { if } \mu \in[p, 2 p-1], \\ p c & \text { if } \mu \in[-(p-1), 0),\end{cases}
$$

where $\sim$ indicates multiplicative congruence modulo units. We also record for completeness that for $n \in \mathbf{Z}$

$$
\gamma_{p}(n, n)= \begin{cases}1 & \text { if } n \leq 0 \\ p & \text { if } n>0\end{cases}
$$

Therefore, the meromorphic function $\gamma_{p}(b, c)$ has a zero if and only if $b \in \mathbf{Z}_{>0}$ and $c \in \mathbf{Z}_{\leq 0}$, while it has a pole if and only if $c \in \mathbf{Z}_{>0}$ and $b \in \mathbf{Z}_{\leq 0}$.

2. Frobenius: comparison between the Results of [GHF] And of [LDE]

We generalize $g(x)$ to

$$
g(x, \lambda)=x_{3}\left(x_{1}+x_{2}\right)+x_{4}\left(\lambda x_{1}+x_{2}\right)=x_{1}\left(x_{3}+\lambda x_{4}\right)+x_{2}\left(x_{3}+x_{4}\right)
$$

and recall first the general notation of $[\mathrm{GHF}]$. The Frobenius map

$$
\alpha_{\vec{a}, \vec{b}, \lambda}^{*}: \mathcal{K}_{\vec{b}, \lambda^{p}}^{\prime} \longrightarrow \mathcal{K}_{\vec{a}, \lambda}^{\prime}
$$

is defined under some mild conditions on $(\vec{a}, \vec{b}, \lambda) \in H\left(\mathbf{C}_{p}\right) \times H\left(\mathbf{C}_{p}\right) \times \mathbf{C}_{p}, p \vec{b}-\vec{a}=$ $\vec{\mu} \in H(\mathbf{Z})$, which we do not state explicitly [GHF, Lemma 4.5.1]. We consider instead the following subregions of $H\left(\mathbf{C}_{p}\right)$, indexed by $\vec{\mu} \in H(\mathbf{Z})$, and of $\mathbf{C}_{p}$, indexed by $\epsilon \geq 0$ :

\section{Definition 2.3.}

$$
\begin{gathered}
\mathcal{H}_{\vec{\mu}}=\left\{\vec{a} \in H\left(\mathbf{C}_{p}\right) \mid d(\vec{a},-\vec{\mu}) \leq p^{-1}\right\}, \\
\left.\mathcal{H}=\bigcup_{\vec{\mu} \in H(\mathbf{Z})} \mathcal{H}_{\vec{\mu}}=\left\{\vec{a} \in H\left(\mathbf{C}_{p}\right)\right) \mid d(\vec{a}, H(\mathbf{Z})) \leq p^{-1}\right\}, \\
S_{\epsilon}=\left\{\lambda \in \mathbf{C}_{p} \mid \epsilon \geq \operatorname{ord}(\lambda) \geq 0=\operatorname{ord}(\lambda-1)\right\}, \\
S=D\left(0,1^{+}\right) \backslash D\left(1,1^{-}\right)=\left\{\lambda \in \mathbf{C}_{p} \mid \operatorname{ord}(\lambda) \geq 0=\operatorname{ord}(\lambda-1)\right\}, \\
D=D\left(0,1^{-}\right)=\left\{\lambda \in \mathbf{C}_{p} \mid \operatorname{ord}(\lambda)>0\right\} .
\end{gathered}
$$

The following lemma is easily checked.

Lemma 2.4. Let

$$
\begin{aligned}
& \mathcal{S}=\left\{\vec{\mu} \in H(\mathbf{Z}) \mid-p<\mu_{i}<p, i=1,2,3,4,\right.\left.\mid-\mu-\mu_{1}-\mu_{3}<p\right\} . \\
& \mu_{1} \geq 0, \mu_{4} \geq 0,0 \leq \mu_{4}-\mu_{2}=
\end{aligned}
$$

Then, if $\vec{\mu}, \vec{\mu}^{\prime} \in \mathcal{S}, \vec{\mu} \neq \vec{\mu}^{\prime}$,

$$
\mathcal{H}_{\vec{\mu}} \cap \mathcal{H}_{\vec{\mu}^{\prime}}=\emptyset
$$


and

$$
\mathcal{H}=\bigcup_{\vec{\mu} \in \mathcal{S}} \mathcal{H}_{\vec{\mu}}
$$

We know from [GHF, Lemma 4.5.1] that $\alpha_{\vec{a}, \vec{b}, \lambda}^{*}=\alpha_{\vec{a}, \frac{\vec{a}+\vec{\mu}}{p}, \lambda}^{*}$ is defined for $(\vec{a}, \lambda) \in$ $\mathcal{H}_{\vec{\mu}} \times S_{\epsilon}$, for any $\vec{\mu} \in H(\mathbf{Z}), 0 \leq \epsilon \leq p^{-1}\left(1-\frac{1}{p}-\frac{1}{p-1}\right)$ and

$$
a_{i}, b_{i} \notin \mathbf{Z}, \text { for } i=1,2,3,4 \text {. }
$$

(The last non-resonance condition is unnecessary for the existence of $\alpha_{\vec{a}, \vec{b}, \lambda}^{*}[\mathrm{~A}-\mathrm{D}]$ ).

We recall the matrix representation ( $c f . \$ 4.7$ of $[\mathrm{GHF}]$; a weakened form of condition (2.5), namely condition (3.1) below, is here necessary)

$$
\alpha_{\vec{a}, \vec{b}, \lambda}^{*}\left([1]_{\vec{b}, \lambda^{p}}^{*},\left[\pi x_{2} x_{3}\right]_{\vec{b}, \lambda^{p}}^{*}\right)=\left([1]_{\vec{a}, \lambda}^{*},\left[\pi x_{2} x_{3}\right]_{\vec{a}, \lambda}^{*}\right) \gamma_{g}(\vec{a}, \vec{b} ; \lambda) .
$$

We also write

$$
\gamma_{g}(\vec{a}, \vec{b} ; \lambda)=\left(\begin{array}{cc}
\gamma_{11}(\vec{a}, \vec{b} ; \lambda) & \gamma_{12}(\vec{a}, \vec{b} ; \lambda) \\
\gamma_{21}(\vec{a}, \vec{b} ; \lambda) & \gamma_{22}(\vec{a}, \vec{b} ; \lambda)
\end{array}\right)=\left(\begin{array}{cc}
\gamma_{11} & \gamma_{12} \\
\gamma_{21} & \gamma_{22}
\end{array}\right)
$$

The results of [GHF, Th. 4.7.1], strengthened in [A-D, Th. 3.3], assert that for a given $\vec{\mu} \in H(\mathbf{Z})$, there exist a polynomial $P(z) \in \mathbf{Z}\left[z_{1}, z_{2}, z_{3}, z_{4}\right]$ and a natural number $N$ such that $P(\vec{a}) \lambda^{N} \gamma_{g}\left(\vec{a}, \frac{\vec{a}+\vec{\mu}}{p} ; \lambda\right)$ extends to an analytic function of $(\vec{a}, \lambda)$ in $\mathcal{H}_{\vec{\mu}} \times S$. By equation (5.40) of [GHF] one may check that the order of the pole of $\gamma_{g}\left(\vec{a}, \frac{\vec{a}+\vec{\mu}}{p} ; \lambda\right)$ at $\lambda=0$ is bounded by $\sup \left(0, \mu_{3}-\mu_{1}\right)$. If we insist that $\mu_{i} \leq p-1$ for $i=1,2,3,4$, then by [GHF, Prop. 6.13 .2 and $\S 13.2$ ] we may take $P(z)=1$ in the previous assertion.

We are especially interested in estimates for the Frobenius matrix similar to those of Chapter 6 of [LDE], but based on formula (4.40) of [GHF]. We will discuss them after having compared the results of $[\mathrm{GHF}]$ and [LDE] as far as the Frobenius structure is concerned.

The theory of [LDE] is related to that of [GHF] by the so-called Laplace transform (Chapters 10 and 11 of [GHF]). This may be done in 2 ways: with respect to the variables $\left(x_{1}, x_{2}\right)$ and with respect to the variables $\left(x_{3}, x_{4}\right)$.

We define

$$
\begin{aligned}
\mathcal{H}_{\vec{\nu}}^{\prime} & =\left\{(a, b, c) \in \mathbf{C}_{p}^{3} \mid d((a, b, c),-\vec{\nu}) \leq p^{-1}\right\}, \text { for } \vec{\nu} \in \mathbf{Z}^{3}, \\
\mathcal{S}^{\prime} & =\left\{\vec{\nu}=\left(\nu_{1}, \nu_{2}, \nu_{3}\right) \mid \nu_{i} \in\{0, \ldots, p-1\}, i=1,2,3\right\}, \\
\mathcal{H}^{\prime} & =\left\{(a, b, c) \in \mathbf{C}_{p}^{3} \mid d\left((a, b, c), \mathbf{Z}^{3}\right) \leq p^{-1}\right\}=\bigcup_{\vec{\nu} \in \mathcal{S}^{\prime}} \mathcal{H}_{\vec{\nu}}^{\prime} .
\end{aligned}
$$

We first consider the algebraic Laplace transform with respect to the variables $\left(x_{1}, x_{2}\right)$

$$
\begin{gathered}
R=\mathbf{C}_{p}(\lambda)\left[x_{1} x_{3}, x_{1} x_{4}, x_{2} x_{3}, x_{2} x_{4}\right] \longrightarrow \tilde{\mathbf{L}}=\mathbf{C}_{p}(\lambda)\left[x, \frac{1}{x(1+x)(1+\lambda x)}\right], \\
x^{u} \longmapsto \frac{x_{3}^{u_{3}} x_{4}^{u_{4}}}{\left(x_{3}+\lambda x_{4}\right)^{u_{1}}\left(x_{3}+x_{4}\right)^{u_{2}}} \frac{\left(a_{1}\right)_{u_{1}}\left(a_{2}\right)_{u_{2}}}{(-\pi)^{u_{1}+u_{2}}}=\frac{x^{u_{4}}}{(1+\lambda x)^{u_{1}}(1+x)^{u_{2}}} \frac{\left(a_{1}\right)_{u_{1}}\left(a_{2}\right)_{u_{2}}}{(-\pi)^{u_{1}+u_{2}}} .
\end{gathered}
$$


In the previous equation we put $\frac{x_{4}}{x_{3}}=x$. Let

$$
x \tilde{f}=x^{a_{4}}(1+x)^{-a_{2}}(1+\lambda x)^{-a_{1}}=x^{b}(1+x)^{c-b}(1+\lambda x)^{-a}
$$

and

$$
x f=x^{b}(1-x)^{c-b}(1-\lambda x)^{-a} .
$$

We define

$$
\mathbf{W}_{f}=\mathbf{L} /(x f)^{-1} \circ x \frac{\partial}{\partial x} \circ x f \mathbf{L}, \quad \tilde{\mathbf{W}}_{\tilde{f}}=\tilde{\mathbf{L}} /(x f)^{-1} \circ x \frac{\partial}{\partial x} \circ x f \tilde{\mathbf{L}} .
$$

For $\lambda$ transcendental over $\mathbf{C}_{p}$, we obtain isomorphic $\mathbf{C}_{p}(\lambda) / \mathbf{C}_{p^{-}}$-differential modules via

$$
\mathbf{L} \longrightarrow \tilde{\mathbf{L}}, \quad g(x) \longmapsto g(-x)
$$

The space considered in $[\mathrm{LDE}]$ is $\mathbf{W}_{f}$. If $(A, B, C) \in \mathbf{C}_{p}^{3}$ are such that

$$
(p A-a, p B-b, p C-c)=\left(\mu_{a, A}, \mu_{b, B}, \mu_{c, C}\right) \in \mathbf{Z}^{3}
$$

and

$$
\begin{gathered}
x h=x^{B}(1-x)^{C-B}(1-\lambda x)^{-A}, \\
x \tilde{h}=x^{B}(1+x)^{C-B}(1+\lambda x)^{-A}, \\
G_{f, h}(x, \lambda)=\frac{x f(x, \lambda)}{x^{p} h\left(x^{p}, \lambda^{p}\right)}, \quad G_{\tilde{f}, \tilde{h}}(x, \lambda)=\frac{x \tilde{f}(x, \lambda)}{x^{p} \tilde{h}\left(x^{p}, \lambda^{p}\right)},
\end{gathered}
$$

then

$$
G_{\tilde{f}, \tilde{h}}(x, \lambda)=(-1)^{\mu_{b, B}} G_{f, h}(-x, \lambda) .
$$

By [GHF, Lemma 11.1], if $a_{1}, a_{2} \notin \mathbf{Z}$, the algebraic Laplace isomorphism induces isomorphisms of algebraic cohomology spaces:

$$
\begin{array}{ccccc}
\mathcal{W}_{\vec{a}}^{\prime} & \stackrel{\sim}{\longrightarrow} \tilde{\mathbf{W}}_{\tilde{f}} & \stackrel{\sim}{\longmapsto} & \mathbf{W}_{f} \\
{[1]} & \longmapsto & {[1]} & \longmapsto & {[1]} \\
{\left[\pi x_{2} x_{3}\right]} & \longmapsto & -a_{2}\left[\frac{1}{1+x}\right] & \longmapsto & -a_{2}\left[\frac{1}{1-x}\right]
\end{array}
$$

In particular, a $\mathbf{C}_{p}(\lambda)$-basis of $\tilde{\mathbf{W}}_{\tilde{f}}$ is given by $\left([1],\left[\frac{1}{1+x}\right]\right)$.

We now set

$$
a=a_{1}, \quad b=a_{4}, \quad c=a_{4}-a_{2}=a_{1}-a_{3},
$$




$$
A=b_{1}, \quad B=b_{4}, C=b_{4}-b_{2}=b_{1}-b_{3},
$$

so that

$$
\mu_{a, A}=\mu_{1}, \quad \mu_{b, B}=\mu_{4}, \quad \mu_{c, C}=\mu_{4}-\mu_{2}=\mu_{1}-\mu_{3} .
$$

So the Laplace transform with respect to the variables $\left(x_{1}, x_{2}\right)$ leads us to consider, via (2.16.1), a linear isomorphism

$$
T_{I}: H\left(\mathbf{C}_{p}\right) \longrightarrow\left(\mathbf{C}_{p}\right)^{3}
$$

(such that $T_{I}(H(\mathbf{Z}))=(\mathbf{Z})^{3}$ and $\left.T_{I}(\mathcal{S})=\mathcal{S}^{\prime}\right)$, and, for any $\vec{\mu} \in H(\mathbf{Z})$, an analytic isomorphism

$$
\begin{aligned}
T_{I}: \mathcal{H}_{\vec{\mu}} & \longmapsto \mathcal{H}_{T_{I}(\vec{\mu})}^{\prime}, \\
\left(a_{1}, a_{2}, a_{3}, a_{4}\right) & \longmapsto(a, b, c)=\left(a_{1}, a_{4}, a_{4}-a_{2}\right) .
\end{aligned}
$$

From [LDE] we know the existence of dual maps, defined for a specialized $\lambda \in S \backslash\{0\}$ and

$$
a, b, c \in \mathbf{Q} \cap \mathbf{Z}_{p}
$$

such that

$$
a, b, a-c, b-c \notin \mathbf{Z},
$$

namely

$$
\bar{\alpha}_{f, h}: \mathbf{W}_{f, \lambda} \longrightarrow \mathbf{W}_{h, \lambda^{p}}
$$

and

$$
\alpha_{f, h}^{*}: \mathbf{K}_{h, \lambda^{p}} \longrightarrow \mathbf{K}_{f, \lambda} .
$$

We observe that conditions $(2.18 .1,2)$ are equivalent, via (2.17), to

$$
a_{i} \in \mathbf{Q} \cap \mathbf{Z}_{p} \backslash \mathbf{Z}, \text { for } i=1,2,3,4 .
$$

If also (2.6.1) is defined, we get commutative diagrams

$$
\begin{array}{rcccc} 
& \mathcal{W}_{\vec{a}, \lambda}^{\prime} & \stackrel{\sim}{\longrightarrow} & \mathbf{W}_{f, \lambda} & \\
\alpha_{\vec{a}, \vec{b}, \lambda} & \downarrow & & \downarrow & (-1)^{\mu_{4}} \gamma_{p}\left(a_{1}, b_{1}\right) \gamma_{p}\left(a_{2}, b_{2}\right) \bar{\alpha}_{f, h} \\
& \mathcal{W}_{\vec{b}, \lambda^{p}}^{\prime} & \stackrel{\sim}{\longrightarrow} & \mathbf{W}_{h, \lambda^{p}} &
\end{array}
$$

Dually, and under the same assumptions (2.18.5), we have the commutative diagrams

$$
\begin{array}{rcccc} 
& \mathbf{K}_{f, \lambda} & \stackrel{\sim}{\longrightarrow} \mathcal{K}_{\vec{a}, \lambda}^{\prime} \\
(-1)^{\mu_{4}} \gamma_{p}\left(a_{1}, b_{1}\right) \gamma_{p}\left(a_{2}, b_{2}\right) \alpha_{f, h}^{*} & \uparrow & & \uparrow \alpha_{\vec{a}, \vec{b}, \lambda}^{*} \\
& \mathbf{K}_{h, \lambda^{p}} \stackrel{\sim}{\longrightarrow} \mathcal{K}_{\vec{b}, \lambda^{p}}^{\prime}
\end{array}
$$


Then, while in the notation of [GHF] we have (2.6.1), in that of [LDE, 4.5.1] (upon renaming $B$ as $\mathbf{B}$ ) we have

$$
\alpha_{f, h}^{*}\left([1]^{*},\left[\frac{1}{1-x}\right]^{*}\right)=\left([1]^{*},\left[\frac{1}{1-x}\right]^{*}\right) \mathbf{B}^{t}=\left([1]^{*},\left[\frac{1}{1-x}\right]^{*}\right)\left(\begin{array}{ll}
\mathbf{B}_{1} & \mathbf{B}_{3} \\
\mathbf{B}_{2} & \mathbf{B}_{4}
\end{array}\right),
$$

for $\lambda \in S \backslash\{0\}$ and $(a, b, c) \in \mathcal{H}^{\prime}$, provided conditions (2.18.1,2) hold. We then have

$$
\left(\begin{array}{ll}
\mathbf{B}_{1} & \mathbf{B}_{2} \\
\mathbf{B}_{3} & \mathbf{B}_{4}
\end{array}\right)=(-1)^{\mu_{4}} \gamma_{p}\left(a_{1}, b_{1}\right)^{-1} \gamma_{p}\left(a_{2}, b_{2}\right)^{-1}\left(\begin{array}{cc}
\gamma_{11} & -\frac{\gamma_{21}}{a_{2}} \\
-b_{2} \gamma_{12} & \frac{b_{2} \gamma_{22}}{a_{2}}
\end{array}\right) .
$$

We now use formula (2.21) to extend, for fixed $\vec{\mu} \in H(\mathbf{Z})$, the matrix $\mathbf{B}$ to a meromorphic function of $(\vec{a}, \lambda) \in \mathcal{H}_{\vec{\mu}} \times S_{\epsilon}$, where $\vec{a}$ stands for $\left(a_{1}, a_{2}, a_{3}, a_{4}\right)$, and $\epsilon \geq 0$ is small, or of $(a, b, c, \lambda)$ in the region $\mathcal{H}_{T_{I}(\vec{\mu})}^{\prime} \times S_{\epsilon}$. We will take the liberty of writing indifferently $\mathbf{B}=\mathbf{B}(\vec{a}, \vec{b} ; \lambda)=\mathbf{B}(a, b, c ; A, B, C ; \lambda)(=\mathbf{B}(\vec{a}, \lambda)=\mathbf{B}(a, b, c ; \lambda))$ according to the context. When we do so, we understand, unless otherwise specified, that we are using the transformation $T_{I}$ (and that $\vec{\mu}$ is somehow specified). We will investigate in the next section the maximal meromorphic extension of the previous functions in $\mathcal{H}_{\vec{\mu}} \times S$.

Remark 2.21.1. The assumption $a, b, c \in \mathbf{Q} \cap \mathbf{Z}_{p}$ is needed in [LDE] to prove that the algebraic cohomology space $\mathbf{W}_{f}$ (and its dual $\mathbf{K}_{f}$ ), for a specialized $\lambda \neq 0,1, \infty$, is isomorphic to its Monsky-Washnitzer counterpart $\mathbf{W}_{f, \lambda}\left(\right.$ resp. $\left.\mathbf{K}_{f, \lambda}\right)(c f$. Chaps. 14 of [LDE]). But the theory of [GHF], where only the dual spaces $\mathcal{K}_{\vec{a}, \lambda}^{\prime}$ are effectively used, is independent of non-Liouville-type assumptions on the $a_{i}$ 's. This permits us to use diagram (2.19.2) freely for $(a, b, c) \in \mathcal{H}^{\prime}$, under only the assumption (2.18.2) (corresponding to (2.5) via $T_{I}$ ), where $\mathbf{K}_{f, \lambda}, \mathbf{K}_{h, \lambda}$ now indicate the algebraic dual cohomology spaces, for a specialized $\lambda \neq 0,1, \infty$. This seems to suggest that more generally the dual algebraic cohomology spaces of Dwork should be endowed with a Frobenius action, even when they do not coincide with their analytic counterpart, as defined in chapter 4 of [LDE]. We finally point out that the map

$$
\mathbf{K}_{f, \lambda} \stackrel{\sim}{\longrightarrow} \mathcal{K}_{\vec{a}, \lambda}^{\prime}
$$

of diagram (2.19.2), "transpose of Laplace transform", in a purely algebraic or in an analytic setting, does not appear in the literature.

The Laplace transform with respect to the variables $\left(x_{3}, x_{4}\right)$ would yield analogous results with

$$
\begin{aligned}
& a=a_{4}, \quad b=a_{1}, \quad c=a_{1}-a_{3}=a_{4}-a_{2}, \\
& A=b_{4}, \quad B=b_{1}, \quad C=b_{1}-b_{3}=b_{4}-b_{2},
\end{aligned}
$$

so that

$$
\mu_{a, A}=\mu_{4}, \quad \mu_{b, B}=\mu_{1}, \quad \mu_{c, C}=\mu_{1}-\mu_{3}=\mu_{4}-\mu_{2} .
$$

As before we get, via (2.22.1), a linear isomorphism

$$
\begin{aligned}
T_{I I}: \mathcal{H}_{\vec{\mu}} & \longrightarrow \mathcal{H}_{T_{I}(\vec{\mu})}^{\prime}, \\
\left(a_{1}, a_{2}, a_{3}, a_{4}\right) & \longmapsto(a, b, c)=\left(a_{4}, a_{1}, a_{4}-a_{2}\right) .
\end{aligned}
$$


(such that $T_{I I}(H(\mathbf{Z}))=(\mathbf{Z})^{3}$ and $\left.T_{I I}(\mathcal{S})=\mathcal{S}^{\prime}\right)$, and, for any $\vec{\mu} \in H(\mathbf{Z})$, an analytic isomorphism

$$
T_{I I}: \mathcal{H}_{\vec{\mu}} \longrightarrow \mathcal{H}_{T_{I I}(\vec{\mu})}^{\prime}
$$

We then obtain commutative diagrams

$$
\begin{array}{rcccc} 
& \mathcal{W}_{\vec{a}, \lambda}^{\prime} & \stackrel{\sim}{\longrightarrow} & \mathbf{W}_{f, \lambda} & \\
\alpha_{\vec{a}, \vec{b}, \lambda} & \downarrow & & \downarrow & (-1)^{\mu_{1}} \gamma_{p}\left(a_{3}, b_{3}\right) \gamma_{p}\left(a_{4}, b_{4}\right) \bar{\alpha}_{f, h} \\
& \mathcal{W}_{\vec{b}, \lambda^{p}}^{\prime} & \stackrel{\sim}{\longrightarrow} & \mathbf{W}_{h, \lambda^{p}} &
\end{array}
$$

and

$$
\begin{aligned}
& \mathcal{K}_{\vec{a}, \lambda}^{\prime} \stackrel{\sim}{\longrightarrow} \mathbf{K}_{f, \lambda} \\
& \alpha_{\vec{a}, \vec{b}, \lambda}^{*} \quad \uparrow \quad \uparrow \quad(-1)^{\mu_{1}} \gamma_{p}\left(a_{3}, b_{3}\right) \gamma_{p}\left(a_{4}, b_{4}\right) \alpha_{f, h}^{*} \\
& \mathcal{K}_{\vec{b}, \lambda^{p}}^{\prime} \stackrel{\sim}{\longrightarrow} \mathbf{K}_{h, \lambda^{p}}
\end{aligned}
$$

As before, for fixed $\vec{\mu} \in H(\mathbf{Z})$, the matrix $\mathbf{B}$ could be viewed as a function of $(\vec{a}, \lambda) \in \mathcal{H}_{\vec{\mu}} \times S_{\epsilon}$, where $\vec{a}$ stands for $\left(a_{1}, a_{2}, a_{3}, a_{4}\right)$, and $\epsilon>0$ is small, or of $(a, b, c, \lambda)$, in the region $\mathcal{H}_{T_{I I}(\vec{\mu})}^{\prime} \times S_{\epsilon}$, via the transformation $T_{I I}$. We then have

$$
\left(\begin{array}{ll}
\mathbf{B}_{1} & \mathbf{B}_{2} \\
\mathbf{B}_{3} & \mathbf{B}_{4}
\end{array}\right)=(-1)^{\mu_{1}} \gamma_{p}\left(a_{3}, b_{3}\right)^{-1} \gamma_{p}\left(a_{4}, b_{4}\right)^{-1}\left(\begin{array}{cc}
\gamma_{11} & -\frac{\gamma_{21}}{a_{3}} \\
-b_{3} \gamma_{12} & \frac{b_{3} \gamma_{22}}{a_{3}}
\end{array}\right)
$$

We now point out that the positions (2.17) and (2.23) define a commutative diagram

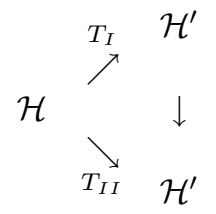

where the vertical arrow interchanges $a$ and $b$, keeping $c$ fixed. From (2.21) and (2.26) we obtain the following formula, valid for any $\vec{\nu}=\left(\mu_{a, A}, \mu_{b, B}, \mu_{c, C}\right) \in \mathbf{Z}^{3}$, $(a, b, c) \in \mathcal{H}_{\vec{\nu}}^{\prime}$, and $(A, B, C)=p^{-1}((a, b, c)+\vec{\nu})$ :

$$
\begin{aligned}
\mathbf{B}(b, a, c ; B, A, C ; \lambda)= & (-1)^{\mu_{a, A}-\mu_{b, B}} \frac{\gamma_{p}(a, A) \gamma_{p}(b-c, B-C)}{\gamma_{p}(b, B) \gamma_{p}(a-c, A-C)} \\
& \cdot\left(\begin{array}{cc}
1 & 0 \\
0 & \frac{C-A}{C-B}
\end{array}\right) \mathbf{B}(a, b, c ; A, B, C ; \lambda)\left(\begin{array}{cc}
1 & 0 \\
0 & \frac{c-b}{c-a}
\end{array}\right) .
\end{aligned}
$$

Formula (2.28) is proved by two different methods in section 5 of $[\mathrm{Ku}]$.

We may specialize the results of this section to the situation considered in $\S 1$, that is, $a_{3}=a_{4}, b_{3}=b_{4}, \mu_{1}$ even. More precisely: $\vec{\mu} \in L(\mathbf{Z}), \mu_{1}$ even, $\vec{a} \in \mathcal{H}_{\vec{\mu}}, \vec{a}$, $\vec{b}=\frac{\vec{a}+\vec{\mu}}{p} \in L\left(\mathbf{C}_{p}\right)$. 
We first use $T_{I}$. The previous assumptions then correspond for $(a, b, c)$ to assuming $c=a-b, C=A-B$ and $\mu_{a, A}$ even. We obtain, for $(a, b, a-b) \in \mathcal{H}^{\prime}$ and $p(A, B, A-B)-(a, b, a-b)=\left(\mu_{a, A}, \mu_{b, B}, \mu_{a, A}-\mu_{b, B}\right) \in \mathbf{Z}^{3}, \mu_{a, A}$ even:

$$
\mathbf{B}(a, b, a-b ; A, B, A-B ;-1)=\left(\begin{array}{cc}
\tau_{1}(a, b ; A, B) & * \\
0 & \tau_{2}(a, b ; A, B)
\end{array}\right),
$$

where

$$
\begin{aligned}
\tau_{1}(a, b ; A, B) & =(-1)^{\mu_{b, B}-\frac{\mu_{a, A}}{2}} \frac{\gamma_{p}\left(\frac{a}{2}, \frac{A}{2}\right) \gamma_{p}\left(b-\frac{a}{2}, B-\frac{A}{2}\right) \gamma_{p}(b, B)}{\gamma_{p}(a, A) \gamma_{p}(2 b-a, 2 B-A)} \\
& =(-1)^{\frac{\mu_{2}}{2}} \frac{\gamma_{p}\left(\frac{a_{1}}{2}, \frac{b_{1}}{2}\right) \gamma_{p}\left(\frac{a_{2}}{2}, \frac{b_{2}}{2}\right) \gamma_{p}\left(a_{3}, b_{3}\right)}{\gamma_{p}\left(a_{1}, b_{1}\right) \gamma_{p}\left(a_{2}, b_{2}\right)}
\end{aligned}
$$

$$
\begin{aligned}
\tau_{2}(a, b ; A, B) & =(-1)^{\mu_{b, B}-\frac{\mu_{a, A}}{2}+\frac{p-1}{2}} \frac{\gamma_{p}\left(\frac{a+1}{2}, \frac{A+1}{2}\right) \gamma_{p}\left(b-\frac{a}{2}+\frac{1}{2}, B-\frac{A}{2}+\frac{1}{2}\right) \gamma_{p}(b, B)}{\gamma_{p}(a, A) \gamma_{p}(1+2 b-a, 1+2 B-A)} \\
& =(-1)^{\frac{\mu_{2}+p-1}{2}} \frac{\gamma_{p}\left(\frac{a_{1}+1}{2}, \frac{b_{1}+1}{2}\right) \gamma_{p}\left(\frac{a_{2}+1}{2}, \frac{b_{2}+1}{2}\right) \gamma_{p}\left(a_{3}, b_{3}\right)}{\gamma_{p}\left(a_{1}, b_{1}\right) \gamma_{p}\left(a_{2}+1, b_{2}+1\right)}
\end{aligned}
$$

where $\left(a_{1}, a_{2}, a_{3}, a_{4}\right)$ and $(a, b, c)$ (resp. $\left(b_{1}, b_{2}, b_{3}, b_{4}\right)$ and $\left.(A, B, C)\right)$ are related via $T_{I}$. We point out that if we assume $\mu_{a, A}, \mu_{b, B} \in\{0, \ldots, p-1\}$, then the entries of the matrix

$$
\left(\begin{array}{cc}
\tau_{1}(a, b ; A, B) & 0 \\
0 & \tau_{2}(a, b ; A, B)
\end{array}\right)
$$

have the same $p$-adic orders as those of the matrix given in the following table:

$$
\begin{aligned}
\left(\begin{array}{ll}
1 & 0 \\
0 & 1
\end{array}\right) & \text { if, }-(p-1) \leq 2 \mu_{b, B}-\mu_{a, A} \leq 0 \\
\left(\begin{array}{cc}
1 & 0 \\
0 & p(2 B-A)
\end{array}\right) & \text { if } 0<2 \mu_{b, B}-\mu_{a, A} \leq p-1, \\
\left(\begin{array}{cc}
p(2 B-A-1) & 0 \\
0 & p(2 B-A)
\end{array}\right) & \text { if } p-1<2 \mu_{b, B}-\mu_{a, A} \leq 2(p-1) .
\end{aligned}
$$

(To check the last statement, recall that $\mu_{a, A}$ is even, so that $2 \mu_{b, B}-\mu_{a, A} \neq p$.)

In our applications, we will be especially interested in the formula

$$
\begin{aligned}
& \mathbf{B}(a, b, 1+a-b ; A, B, 1+A-B ;-1) \\
& \quad=\left(\begin{array}{cc}
0 & \frac{1-B}{4 B-2 A} \\
1 & \frac{4 B-2 A-1}{4 B-2 A}
\end{array}\right) \mathbf{B}(a, b, a-b ; A, B, A-B ;-1)\left(\begin{array}{cc}
\frac{4 b-2 a-1}{b-1} & 1 \\
\frac{4 b-2 a}{1-b} & 0
\end{array}\right)
\end{aligned}
$$


that is easily deduced from [Dw1, (3.0.3)], where the matrix $\gamma$ that is used is the transpose of our B. As a consequence

$$
\begin{aligned}
& \mathbf{B}(a, b, 1+a-b ; A, B, 1+A-B ;-1) \\
&=\left(\begin{array}{cc}
\frac{1-B}{1-b} \frac{2 b-a}{2 B-A} \tau_{2}(a, b ; A, B) & 0 \\
* & \tau_{1}(a, b ; A, B)
\end{array}\right),
\end{aligned}
$$

so that

(2.33.1)

$\mathbf{B}_{1}(a, b, 1+a-b ; A, B, 1+A-B ;-1)$

$$
=(-1)^{\mu_{b, B}-\frac{\mu_{a, A}}{2}+\frac{p-1}{2}} \frac{\gamma_{p}\left(\frac{a+1}{2}, \frac{A+1}{2}\right) \gamma_{p}\left(b-\frac{a}{2}+\frac{1}{2}, B-\frac{A}{2}+\frac{1}{2}\right) \gamma_{p}(b-1, B-1)}{\gamma_{p}(a, A) \gamma_{p}(2 b-a, 2 B-A)},
$$

$$
\begin{aligned}
& \mathbf{B}_{4}(a, b, 1+a-b ; A, B, 1+A-B ;-1) \\
& \quad=(-1)^{\mu_{b, B}-\frac{\mu_{a, A}}{2}} \frac{\gamma_{p}\left(\frac{a}{2}, \frac{A}{2}\right) \gamma_{p}\left(b-\frac{a}{2}, B-\frac{A}{2}\right) \gamma_{p}(b, B)}{\gamma_{p}(a, A) \gamma_{p}(2 b-a, 2 B-A)} .
\end{aligned}
$$

In particular, if $0 \leq 2 \mu_{b, B}-\mu_{a, A} \leq p-1$,

$$
\begin{gathered}
\mathbf{B}_{2}(a, b, 1+a-b ; A, B, 1+A-B ;-1)=0, \\
\left|\mathbf{B}_{4}(a, b, 1+a-b ; A, B, 1+A-B ;-1)\right|=1,
\end{gathered}
$$

$$
\left|\mathbf{B}_{1}(a, b, 1+a-b ; A, B, 1+A-B ;-1)\right| \begin{cases}=p^{-1}|B-1| & \text { if } \mu_{b}<p-1 \\ =1 & \text { if } \mu_{b}=p-1\end{cases}
$$

By use of $T_{I I}$ or of (2.28), we similarly obtain, for $(a, b, b-a) \in \mathcal{H}^{\prime}$ and $p(A, B, B-A)-(a, b, b-a)=\left(\mu_{a, A}, \mu_{b, B}, \mu_{b, B}-\mu_{a, A}\right) \in \mathbf{Z}^{3}, \mu_{b, B}$ even:

$$
\mathbf{B}(a, b, b-a ; A, B, B-A ;-1)=\left(\begin{array}{cc}
\bar{\tau}_{1}(a, b ; A, B) & * \\
0 & \bar{\tau}_{2}(a, b ; A, B)
\end{array}\right),
$$

where

$$
\begin{gathered}
\bar{\tau}_{1}(a, b ; A, B)=(-1)^{\frac{\mu_{b, B}}{2}} \frac{\gamma_{p}\left(\frac{b}{2}, \frac{B}{2}\right) \gamma_{p}\left(a-\frac{b}{2}, A-\frac{B}{2}\right)}{\gamma_{p}(a, A)} \\
=(-1)^{\frac{\mu_{1}}{2}} \frac{\gamma_{p}\left(\frac{a_{1}}{2}, \frac{b_{1}}{2}\right) \gamma_{p}\left(\frac{a_{2}}{2}, \frac{b_{2}}{2}\right)}{\gamma_{p}\left(a_{3}, b_{3}\right)}, \\
\bar{\tau}_{2}(a, b ; A, B)=(-1)^{\frac{\mu_{b, B}}{2}+\frac{p-1}{2}} \frac{\gamma_{p}\left(\frac{b+1}{2}, \frac{B+1}{2}\right) \gamma_{p}\left(a-\frac{b}{2}+\frac{1}{2}, A-\frac{B}{2}+\frac{1}{2}\right)}{\gamma_{p}(1+a, 1+A)} \\
=(-1)^{\frac{\mu_{1}+p-1}{2}} \frac{\gamma_{p}\left(\frac{a_{1}+1}{2}, \frac{b_{1}+1}{2}\right) \gamma_{p}\left(\frac{a_{2}+1}{2}, \frac{b_{2}+1}{2}\right)}{\gamma_{p}\left(1+a_{3}, 1+b_{3}\right)},
\end{gathered}
$$


where $\left(a_{1}, a_{2}, a_{3}, a_{4}\right)$ and $(a, b, c)$ (resp. $\left(b_{1}, b_{2}, b_{3}, b_{4}\right)$ and $\left.(A, B, C)\right)$ are related via $T_{I I}$. Again, if we assume $\mu_{a, A}, \mu_{b, B} \in\{0, \ldots, p-1\}$, then the entries of the matrix

$$
\left(\begin{array}{cc}
\bar{\tau}_{1}(a, b ; A, B) & 0 \\
0 & \bar{\tau}_{2}(a, b ; A, B)
\end{array}\right)
$$

have the same $p$-adic orders as those of the matrix given in the following table:

$$
\begin{aligned}
& \left(\begin{array}{cc}
p\left(A-\frac{B}{2}\right) & 0 \\
0 & p A
\end{array}\right) \quad \text { if }-(p-1) \leq 2 \mu_{a, A}-\mu_{b, B}<0, \mu_{a, A} \neq 0, \\
& \left(\begin{array}{cc}
p\left(A-\frac{B}{2}\right) & 0 \\
0 & 1
\end{array}\right) \text { if } \mu_{a, A}=0, \mu_{b, B} \neq 0, \\
& \left(\begin{array}{cc}
1 & 0 \\
0 & p A
\end{array}\right) \quad \text { if } 0 \leq 2 \mu_{a, A}-\mu_{b, B} \leq p-1, \mu_{a, A} \neq 0 \text {, } \\
& \left(\begin{array}{ll}
1 & 0 \\
0 & 1
\end{array}\right) \text { if } p-1<2 \mu_{a, A}-\mu_{b, B} \leq 2(p-1) \text { or } \mu_{a, A}=\mu_{b, B}=0 \text {. }
\end{aligned}
$$

As before, we deduce

$$
\begin{aligned}
& \mathbf{B}(a, b, 1+b-a ; A, B, 1+B-A ;-1) \\
& \quad=\left(\begin{array}{cc}
\frac{1+B-2 A}{1+b-2 a} \frac{a}{A} \bar{\tau}_{2}(a, b ; A, B) & 0 \\
* & \bar{\tau}_{1}(a, b ; A, B)
\end{array}\right),
\end{aligned}
$$

so that

$$
\begin{gathered}
\mathbf{B}_{1}(a, b, 1+b-a ; A, B, 1+B-A ;-1) \\
=(-1)^{\frac{\mu_{b, B}}{2}+\frac{p-1}{2}} \frac{\gamma_{p}\left(\frac{b+1}{2}, \frac{B+1}{2}\right) \gamma_{p}\left(a-\frac{b}{2}-\frac{1}{2}, A-\frac{B}{2}-\frac{1}{2}\right)}{\gamma_{p}(a, A)}, \\
\mathbf{B}_{4}(a, b, 1+b-a ; A, B, 1+B-a ;-1) \\
=(-1)^{\frac{\mu_{b, B}}{2}} \frac{\gamma_{p}\left(\frac{b}{2}, \frac{B}{2}\right) \gamma_{p}\left(a-\frac{b}{2}, A-\frac{B}{2}\right)}{\gamma_{p}(a, A)} .
\end{gathered}
$$

\section{Approximations of the Frobenius matrix $(p \geq 7)$}

We now consider simultaneously the theory of [GHF] and of [LDE] as related by the Laplace transform with respect to $\left(x_{1}, x_{2}\right)$. For $\vec{\mu} \in \mathcal{S}$, we examine the Frobenius matrix $\mathbf{B}(\vec{a} ; \lambda)=\mathbf{B}\left(\vec{a}, \frac{\vec{a}+\vec{\mu}}{p} ; \lambda\right)$ of $(2.20)$ as a function of $(\vec{a}, \lambda) \in \mathcal{H}_{\vec{\mu}} \times S$, and, via $T_{I}$, as a function of $(a, b, c ; \lambda) \in \mathcal{H}_{T_{I}(\vec{\mu})}^{\prime} \times S, T_{I}(\vec{\mu}) \in \mathcal{S}^{\prime}$. We abuse notation on setting $T_{I}(\vec{\mu})=\left(\mu_{a}, \mu_{b}, \mu_{c}\right) \in \mathcal{S}^{\prime}$, and aim at making more precise the classification in terms of $\left(\mu_{a}, \mu_{b}, \mu_{c}\right)$ given in [LDE, Chap. 6]. We are mainly interested in the so-called split cases, namely cases 1 and 2 below.

We subdivide $\mathcal{S}$ (resp. $\mathcal{S}^{\prime}$ ) into 9 disjoint sets: Case 0 :

$$
\mu_{a}=\mu_{b}=\mu_{c}
$$

i.e.

$$
\mu_{1}=\mu_{4}=\mu_{4}-\mu_{2}
$$


Case 1 :

$$
\mu_{c}<\min \left(\mu_{a}, \mu_{b}\right)
$$

i.e.

$$
\mu_{4}-\mu_{2}<\min \left(\mu_{1}, \mu_{4}\right)
$$

Case $1^{\prime}$ :

$$
\mu_{c}=\mu_{a}<\mu_{b}
$$

i.e.

$$
\mu_{4}-\mu_{2}=\mu_{1}<\mu_{4} .
$$

Case $1^{\prime \prime}$ :

$$
\mu_{c}=\mu_{b}<\mu_{a},
$$

i.e.

$$
\mu_{4}-\mu_{2}=\mu_{4}<\mu_{1} .
$$

Case 2 :

$$
\mu_{c}>\max \left(\mu_{a}, \mu_{b}\right),
$$

i.e.

$$
\mu_{4}-\mu_{2}>\max \left(\mu_{1}, \mu_{4}\right) .
$$

Case $2^{\prime}$ :

$$
\mu_{c}=\mu_{b}>\mu_{a},
$$

i.e.

$$
\mu_{4}-\mu_{2}=\mu_{4}>\mu_{1} .
$$

Case $2^{\prime \prime}$ :

$$
\mu_{c}=\mu_{a}>\mu_{b},
$$

i.e.

$$
\mu_{4}-\mu_{2}=\mu_{1}>\mu_{4} .
$$

Case 3 :

$$
\mu_{b}<\mu_{c}<\mu_{a}
$$

i.e.

$$
\mu_{4}<\mu_{4}-\mu_{2}<\mu_{1} .
$$

Case 4 :

$$
\mu_{a}<\mu_{c}<\mu_{b},
$$

i.e.

$$
\mu_{1}<\mu_{4}-\mu_{2}<\mu_{4} .
$$

We observe that another characterization of the previous cases is Case 0 :

$$
\mu_{2}=0, \mu_{3}=0
$$

Case 1 :

$$
\mu_{2}>0, \mu_{3}>0
$$

Case $1^{\prime}$ :

$$
\mu_{2}>0, \mu_{3}=0 \text {. }
$$


Case 1" :

$$
\mu_{2}=0, \mu_{3}>0 \text {. }
$$

Case 2 :

$$
\mu_{2}<0, \mu_{3}<0 \text {. }
$$

Case $2^{\prime}$ :

$$
\mu_{2}=0, \mu_{3}<0 .
$$

Case $2^{\prime \prime}$ :

$$
\mu_{2}<0, \mu_{3}=0 \text {. }
$$

Case 3 :

$$
\mu_{2}<0, \mu_{3}>0 \text {. }
$$

Case 4 :

$$
\mu_{2}>0, \mu_{3}<0 .
$$

We fix a constant $\epsilon, 0 \leq \epsilon \leq p^{-1}\left(1-\frac{1}{p}-\frac{1}{p-1}\right)$. The results of [LDE, Chap. 24], together with the previously mentioned [GHF, Th. 4.7.1] and [AD, Th. 3.3], ensure that, for $i, j=1,2, \gamma_{i, j}(\vec{a}, \lambda)$, as defined by formulas $(2.6 .1,2)$ for $(\vec{a}, \lambda) \in \mathcal{H}_{\vec{\mu}} \times S_{\epsilon}$ and

$$
a_{i}, b_{i} \notin \mathbf{Z}_{>0}
$$

for $i=1,2,3,4$, extends to an analytic function on $\mathcal{H}_{\vec{\mu}} \times S$ defined over the field $\mathbf{Q}(\pi)$. This matrix does not need to represent the Frobenius map in the sense of formula (2.6.1) for all $(\vec{a}, \lambda) \in \mathcal{H}_{\vec{\mu}} \times S$, in particular because the basis with respect to which that matrix is generically calculated fails to specialize to a basis of $\mathcal{K}_{\vec{a}, \lambda}^{\prime}$, for values of $(\vec{a}, \lambda)$ outside of $\mathcal{H}_{\vec{\mu}} \times S_{\epsilon}$, or when condition (3.1) is violated.

We recall formula (4.40) of [GHF] in our special case, insisting that $\lambda \in S_{\epsilon}$ and that (3.1) holds. We set

$$
\begin{aligned}
& w_{1}=(0,0,0,0), \quad w_{2}=(0,1,1,0), \\
& \hat{\omega}_{1}=1=x^{w_{1}}, \quad \hat{\omega}_{2}=\pi x_{2} x_{3}=\pi^{k\left(w_{2}\right)} x^{w_{2}},
\end{aligned}
$$

which represent a basis of $\mathcal{W}_{\vec{a}, \lambda}^{\prime}$, and

$$
\omega_{i, \vec{a}, \lambda}^{*}=\sum_{\vec{v} \in H(\mathbf{Z})} \Lambda_{i, \vec{v}}(\vec{a}, \lambda) x^{-\vec{v}}
$$

for $i=1,2$, the dual basis of $\mathcal{K}_{\vec{a}, \lambda}^{\prime}$, under the assumption (3.1). According to [GHF, 3.39], we have for $i=1,2$ and $\vec{v} \in H^{+}(\mathbf{Z})$

$$
\Lambda_{i, \vec{v}}(\vec{a}, \lambda) \in(\pi \lambda(1-\lambda))^{-k(\vec{v})} \mathbf{Z}\left[a_{1}, a_{2}, a_{3}, a_{4}, \lambda\right] .
$$


In particular we may compute:

$$
\begin{aligned}
& \Lambda_{1,(0,0,0,0)}(\vec{a}, \lambda)=1, \\
& \Lambda_{1,(0,1,1,0)}(\vec{a}, \lambda)=0 \\
& \Lambda_{1,(1,0,1,0)}(\vec{a}, \lambda)=-\frac{a_{3}}{\pi}, \\
& \Lambda_{1,(1,0,0,1)}(\vec{a}, \lambda)=\frac{a_{3}-a_{1}}{\pi \lambda}, \\
& \Lambda_{1,(0,1,0,1)}(\vec{a}, \lambda)=-\frac{a_{2}}{\pi}, \\
& \Lambda_{2,(0,0,0,0)}(\vec{a}, \lambda)=0 \\
& \Lambda_{2,(0,1,1,0)}(\vec{a}, \lambda)=\frac{1}{\pi} \\
& \Lambda_{2,(1,0,1,0)}(\vec{a}, \lambda)=-\frac{1}{\pi}, \\
& \Lambda_{2,(1,0,0,1)}(\vec{a}, \lambda)=\frac{1}{\pi \lambda} \\
& \Lambda_{2,(0,1,0,1)}(\vec{a}, \lambda)=-\frac{1}{\pi} .
\end{aligned}
$$

Lemma 3.4.1. Let $\chi$ be the step function (i.e. the characteristic function of the subset $(0, \infty)$ of $\mathbf{R})$. For any $\vec{v} \in H^{+}(\mathbf{Z})$, we have

$$
\Lambda_{1, \vec{v}}(\vec{a}, \lambda) \in a_{2}^{\chi\left(v_{2}\right)} a_{2}^{\chi\left(v_{3}\right)}(\pi \lambda(1-\lambda))^{-k(\vec{v})} \mathbf{Z}\left[a_{1}, a_{2}, a_{3}, a_{4}, \lambda\right] .
$$

Proof. When $k(\vec{v})=1$, our result is contained in (3.4). We use induction on $k(\vec{v})$. Let $\vec{v}, \vec{w} \in H^{+}(\mathbf{Z}), k(\vec{v})=d-1, k(\vec{w})=1$. Formula (3.40) of [GHF] reads

$$
\Lambda_{1, \vec{v}+\vec{w}}(\vec{a}, \lambda)=\sum_{j=1}^{2} M(\vec{a}+\vec{w}, \vec{a} ; \lambda)_{j, 1} \Lambda_{j, \vec{v}}(\vec{a}+\vec{w}, \lambda) \pi^{-1},
$$

where the matrices $M(\vec{a}+\vec{w}, \vec{a} ; \lambda)$ are explicitly described in [GHF, 13.2] (see also 1.10.5)). We are left to prove that $M(\vec{a}+\vec{w}, \vec{a} ; \lambda)_{j, 1} \Lambda_{j, \vec{v}}(\vec{a}+\vec{w}, \lambda)$ is divisible by $a_{2}^{\chi\left(v_{2}+w_{2}\right)} a_{3}^{\chi\left(v_{3}+w_{3}\right)}$, for $j=1,2$. Now, $a_{2} a_{3}$ divides $M(\vec{a}+\vec{w}, \vec{a} ; \lambda)_{2,1}$ by [GHF, 13.2]. We are left to consider $M(\vec{a}+\vec{w}, \vec{a} ; \lambda)_{1,1} \Lambda_{1, \vec{v}}(\vec{a}+\vec{w}, \lambda)$. Let $i$ be either 2 or 3 . We may assume that $\chi\left(v_{i}+w_{i}\right)=1$. If $w_{i}=0$, then $\chi\left(v_{i}\right)=1$ and so by hypothesis $\Lambda_{1, \vec{v}}(\vec{a}+\vec{w}, \lambda)$ is divisible by $a_{i}+w_{i}=a_{i}$. Hence we may assume $w_{i} \neq 0$, and it is enough to check that $a_{i}^{\chi\left(w_{i}\right)}$ divides $M(\vec{a}+\vec{w}, \vec{a} ; \lambda)_{1,1}$, if $k(\vec{w})=1$. The possibilities are:

1) $\vec{w}=(0,1,1,0)$. Here $M(\vec{a}+\vec{w}, \vec{a} ; \lambda)_{1,1}=0$.

2) $\vec{w}=(1,0,1,0)$. Here $a_{3}$ divides $M(\vec{a}+\vec{w}, \vec{a} ; \lambda)_{1,1}$.

3) $\vec{w}=(1,0,0,1)$. The assertion is trivial.

4) $\vec{w}=(0,1,0,1)$. Here $a_{2}$ divides $M(\vec{a}+\vec{w}, \vec{a} ; \lambda)_{1,1}$ Q.E.D.

We also put

$$
\theta(t)=\exp \pi\left(t-t^{p}\right)=\sum_{j=0}^{\infty} c_{j} t^{j}
$$




$$
F(x, \lambda)=\theta\left(x_{1} x_{3}\right) \theta\left(x_{2} x_{3}\right) \theta\left(x_{2} x_{4}\right) \theta\left(\lambda x_{1} x_{4}\right)=\sum_{\vec{u} \in H^{+}(\mathbf{Z})} A_{\vec{u}}(\lambda) x^{\vec{u}} .
$$

We recall that

$$
\operatorname{ord} c_{j} \geq \begin{cases}\frac{j}{p-1} & \text { if } j=0, \ldots, p^{2}-1 \\ j \frac{p-1}{p^{2}} & \text { if } j=p^{2}, p^{2}+1, \ldots\end{cases}
$$

Then

$$
\begin{aligned}
\gamma_{j, i}(\vec{a}, \lambda) & =\left\langle\alpha_{\vec{a}, \frac{\vec{a}+\vec{\mu}}{p}, \lambda}\left(\omega_{i, \frac{\vec{a}+\vec{\mu}}{p}, \lambda^{p}}^{*}\right), \pi^{k\left(w_{j}\right)} x^{w_{j}}\right\rangle \\
& =\pi^{k\left(w_{j}\right)} \sum A_{\vec{u}}(\lambda) \Lambda_{i, \vec{v}}\left(\frac{\vec{a}+\vec{\mu}}{p}, \lambda^{p}\right)
\end{aligned}
$$

where the sum runs over the set of $\vec{u}, \vec{v} \in H^{+}(\mathbf{Z})$ such that $\vec{u}=\vec{\mu}-w_{j}+p \vec{v}$, for $i=1,2$. We can now ameliorate, in our special case, formula (4.40) of [GHF]

$$
\begin{aligned}
\operatorname{ord} \pi^{k\left(w_{j}\right)} A_{\vec{u}}(\lambda) \Lambda_{i, \vec{v}}\left(\frac{\vec{a}+\vec{\mu}}{p}, \lambda^{p}\right) & \\
& \geq \begin{cases}\frac{k(\vec{\mu})}{p-1}+k(\vec{v}) & \text { if } k(\vec{v}) \leq p-2, \\
\frac{k(\vec{\mu})}{p-1}+k(\vec{v})\left(1-\frac{1}{p}-\frac{1}{p-1}\right)-\frac{(2 p-1)(2 p-2)}{p^{2}(p-1)} & \text { if } k(\vec{v}) \geq p-1,\end{cases}
\end{aligned}
$$

for all $(\vec{a}, \lambda) \in \mathcal{H}_{\vec{\mu}} \times S_{0}$.

To simplify our coming calculations, we then observe that if $p \geq 7$ and $h=$ $0,1,2,3$, any term $\pi^{k\left(w_{j}\right)} A_{\vec{u}}(\lambda) \Lambda_{i, \vec{v}}\left(\vec{b}, \lambda^{p}\right)$, with $k(\vec{v}) \geq h$, of the sum appearing in (3.7) has in $\mathcal{H}_{\vec{\mu}} \times S_{0}$ at least the $p$-adic order $\frac{k(\vec{\mu})}{p-1}+h$. We will then assume for simplicity from now on that $p \geq 7$. We then evaluate the dominant term $\gamma_{j, i}^{0}$ of $\gamma_{j, i}$, for $i, j=1,2$, in the sense of the supnorm $\|-\|_{\vec{\mu}}$ on $\mathcal{H}_{\vec{\mu}} \times S_{0}$.

Calculation of $\gamma_{1,1}^{0}$ in cases $0,1,1^{\prime}$ and $1^{\prime \prime}$. We take

$$
\gamma_{1,1}^{0}(\vec{a}, \lambda)=A_{\vec{\mu}}(\lambda)
$$

We solve

$$
\left(\mu_{1}, \mu_{2}, \mu_{3}, \mu_{4}\right)=\left(u_{13}+u_{14}, u_{23}+u_{24}, u_{13}+u_{23}, u_{14}+u_{24}\right),
$$

getting

$$
\begin{aligned}
& u_{14}=t, \\
& u_{24}=\mu_{4}-t, \\
& u_{13}=\mu_{1}-t, \\
& u_{23}=\mu_{2}-\mu_{4}+t .
\end{aligned}
$$


So:

$$
\begin{gathered}
\gamma_{1,1}^{0}(\vec{a}, \lambda)=\sum_{t=\mu_{4}-\mu_{2}}^{\min \left(\mu_{1}, \mu_{4}\right)} c_{t} c_{\mu_{4}-t} c_{\mu_{1}-t} c_{\mu_{2}-\mu_{4}+t} \lambda^{t} \\
=\sum_{t=\mu_{4}-\mu_{2}}^{\min \left(\mu_{1}, \mu_{4}\right)} \frac{\pi^{\mu_{1}+\mu_{2}} \lambda^{t}}{t !\left(\mu_{4}-t\right) !\left(\mu_{1}-t\right) !\left(\mu_{2}-\mu_{4}+t\right) !} \\
=\frac{\pi^{\mu_{1}+\mu_{2}}}{\mu_{1} ! \mu_{2} !} \sum_{t=\mu_{4}-\mu_{2}}^{\min \left(\mu_{1}, \mu_{4}\right)}\left(\begin{array}{c}
\mu_{1} \\
t
\end{array}\right)\left(\begin{array}{c}
\mu_{2} \\
\mu_{4}-t
\end{array}\right) \lambda^{t} \\
=\gamma_{p}\left(-\mu_{1}, 0\right) \gamma_{p}\left(-\mu_{2}, 0\right) \sum_{t=\mu_{4}-\mu_{2}}^{\min \left(\mu_{1}, \mu_{4}\right)}\left(\begin{array}{c}
\mu_{1} \\
t
\end{array}\right)\left(\begin{array}{c}
\mu_{2} \\
\mu_{4}-t
\end{array}\right) \lambda^{t} \\
=\left((-1)^{\mu_{4}} \gamma_{p}\left(-\mu_{1}, 0\right) \gamma_{p}\left(-\mu_{2}, 0\right)\right)(-1)^{\mu_{b}} \sum_{s+t=\mu_{b}}\left(\begin{array}{c}
\mu_{b}-\mu_{c} \\
s
\end{array}\right)\left(\begin{array}{c}
\mu_{a} \\
t
\end{array}\right) \lambda^{t} .
\end{gathered}
$$

Taking into account the previous section, this gives precisely the value expected from the calculation of $\chi_{1}$ in [LDE, 6.4.8]. We write the previous result in the form

$$
\gamma_{1,1}^{0}(\vec{a}, \lambda)=\pi^{k(\vec{\mu})} \lambda^{\mu_{c}} P_{1,1}^{\vec{\mu}}(\lambda),
$$

where $P_{1,1}^{\vec{\mu}}(\lambda)$ is a polynomial in $\mathbf{Z}_{p}[\lambda]$ of Gauss norm 1 whose zeros all have absolute value 1 . We know from [LDE, Lemma 24.4] that $\gamma_{1,1}(\vec{a}, \lambda) \lambda^{-\mu_{c}}$ is analytic in $\mathcal{H}_{\vec{\mu}} \times S$. Our calculation following (3.8) then shows that

$$
\left\|\gamma_{1,1}-\gamma_{1,1}^{0}\right\|_{\vec{\mu}}<p^{-\frac{k(\vec{\mu})}{p-1}} .
$$

Let $S^{\vec{\mu}}$ denote the region

$$
S^{\vec{\mu}}=\left\{\lambda \in S|| P_{1,1}^{\vec{\mu}}(\lambda) \mid=1\right\} .
$$

We notice here that $S^{\vec{\mu}}$ is stable under the map $\lambda \longmapsto \lambda^{p}$. We call $S^{\vec{\mu}}$ the Hasse domain of type $\vec{\mu}$. If we are in cases 0 or $1^{\prime \prime}$, then in fact $S^{\vec{\mu}}=S$. In case 0 this definition will conflict with the one given in (3.27), related to the entry $\gamma_{2,2}$. Case 0 is not too interesting for us; rather than introducing a heavier notation, we will agree that a result on $\gamma_{1,1}$ (resp. $\gamma_{2,2}$ ) involves $S^{\vec{\mu}}$ as defined in (3.14) (resp. (3.27)). It is clear that in any case $D \subset S^{\vec{\mu}}$. We conclude that in cases $0,1,1^{\prime}$ and $1^{\prime \prime}$

$$
\gamma_{1,1}(\vec{a}, \lambda)=\lambda^{\mu_{c}} \pi^{k(\vec{\mu})} u_{1,1}(\vec{a}, \lambda)
$$

where $u_{1,1}(\vec{a}, \lambda)$ is an analytic element bounded by 1 on $\mathcal{H}_{\vec{\mu}} \times S$, defined over the field $\mathbf{Q}(\pi)$, which is of constant absolute value 1 all over $\mathcal{H}_{\vec{\mu}} \times S^{\vec{\mu}}$.

Estimates for $\gamma_{1,1}$ in case 2. Formula (3.7) indicates that only terms with $k(\vec{v}) \geq 1$ need to be considered. But in fact, since $\mu_{2}$ and $\mu_{3}$ are both negative, $\vec{v}=$ $(0,1,1,0)$ is the only term with $k(\vec{v})=1$ that may appear. That term gets cancelled, 
since $\Lambda_{1,(0,1,1,0)}(\vec{a}, \lambda)=0$. On the other hand, Lemma 3.4 .1 shows divisibility by $b_{2} b_{3}$. In the end, considering [LDE, Lemma 24.4], we have in case 2

$$
\gamma_{1,1}(\vec{a}, \lambda)=p^{2} \pi^{k(\vec{\mu})} b_{2} b_{3} \lambda^{\mu_{c}} u_{1,1}(\vec{a}, \lambda),
$$

where $u_{1,1}(\vec{a}, \lambda)$ represents an analytic element defined over the field $\mathbf{Q}(\pi)$ and bounded by 1 on $\mathcal{H}_{\vec{\mu}} \times S$.

Estimates for $\gamma_{1,1}$ in cases $2^{\prime}, 2^{\prime \prime}, 3$ and 4 . We put

$$
\gamma_{1,1}^{0}(\vec{a}, \lambda)= \begin{cases}-\frac{b_{2}}{\pi} A_{\vec{\mu}+p(0,1,0,1)}(\lambda) & \text { in cases } 2^{\prime \prime} \text { and } 3, \\ -\frac{b_{3}}{\pi} A_{\vec{\mu}+p(1,0,1,0)}(\lambda) & \text { in cases } 2^{\prime} \text { and } 4 .\end{cases}
$$

We see that

$$
\left\|\gamma_{1,1}^{0}(\vec{a}, \lambda)\right\|_{\vec{\mu}} \leq\left|\pi^{k(\vec{\mu})} p\right| .
$$

Formula (3.8), in which only terms with $k(\vec{v}) \geq 2$ need now to be considered, shows that

$$
\left\|\gamma_{1,1}(\vec{a}, \lambda)-\gamma_{1,1}^{0}(\vec{a}, \lambda)\right\|_{\vec{\mu}} \leq\left|\pi^{k(\vec{\mu})} p^{2}\right| .
$$

In the end, considering [LDE, Lemma 24.4] and lemma 3.4.1, we have in cases $2^{\prime}$, $2^{\prime \prime}, 3$ and 4

$$
\gamma_{1,1}(\vec{a}, \lambda)=p \pi^{k(\vec{\mu})} \lambda^{\mu_{c}} \begin{cases}b_{2} u_{1,1}(\vec{a}, \lambda) & \text { in cases } 2^{\prime \prime}, 3 \\ b_{3} u_{1,1}(\vec{a}, \lambda) & \text { in cases } 2^{\prime}, 4\end{cases}
$$

where $u_{1,1}(\vec{a}, \lambda)$ represents an analytic element defined over the field $\mathbf{Q}(\pi)$ and bounded by 1 on $\mathcal{H}_{\vec{\mu}} \times S$.

Estimates for $\gamma_{2,1}$ in all cases. We refer to formula (3.7), where in any case $\vec{v}-w_{2} \in H^{+}(\mathbf{Z})$. We take

$$
\gamma_{2,1}^{0}(\vec{a}, \lambda)=\left\{\begin{array}{l}
\pi A_{\vec{\mu}-w_{2}}(\lambda) \text { if } \mu_{2}>0 \text { and } \mu_{3}>0 \text { (case 1); } \\
\left.-b_{2} A_{\vec{\mu}-w_{2}+p(0,1,0,1)}(\lambda) \text { if } \mu_{2} \leq 0 \text { and } \mu_{3}>0 \text { (cases } 1^{\prime \prime}, 3\right) ; \\
\left.\pi \sum_{k(\vec{v})=2} A_{\vec{\mu}-w_{2}+p \vec{v}}(\lambda) \Lambda_{1, \vec{v}}\left(\vec{b}, \lambda^{p}\right) \text { if } \mu_{3} \leq 0 \text { (cases } 0,1^{\prime}, 2,2^{\prime}, 2^{\prime \prime}, 4\right) .
\end{array}\right.
$$

We now easily estimate $\left\|\gamma_{2,1}^{0}(\vec{a}, \lambda)\right\|_{\vec{\mu}}$, while, with the help of formula (3.8), we can bound the remaining terms in formula (3.7). We obtain

$$
\left\|\gamma_{2,1}(\vec{a}, \lambda)\right\|_{\vec{\mu}} \leq \begin{cases}\left|\pi^{k(\vec{\mu})}\right| & \text { in case } 1 ; \\ \left|p \pi^{k(\vec{\mu})}\right| & \text { in cases } 1^{\prime \prime} \text { and } 3 ; \\ \left|p^{2} \pi^{k(\vec{\mu})}\right| & \text { in cases } 0,1^{\prime}, 2,2^{\prime}, 2^{\prime \prime}, 4 .\end{cases}
$$


We know from [LDE, Lemma 24.4] that $\gamma_{2,1}(\vec{a}, \lambda) \lambda^{-\mu_{c}-1}$ is analytic in $\mathcal{H}_{\vec{\mu}} \times S$. Lemma 3.4.1 shows that that that analytic function is divisible by $b_{2}$ (resp. $b_{3}$ ) if $\mu_{2}<0$ (resp. $\mu_{3}<0$ ). We then put

$$
\gamma_{2,1}(\vec{a}, \lambda)=\lambda^{\mu_{c}+1} u_{2,1}(\vec{a}, \lambda) \begin{cases}\pi^{k(\vec{\mu})} & \text { in cases } 0,1,1^{\prime}, 1^{\prime \prime}, \\ p^{2} \pi^{k(\vec{\mu})} b_{2} b_{3} & \text { in case 2, } \\ p \pi^{k(\vec{\mu})} b_{2} & \text { in cases } 2^{\prime \prime}, 3, \\ p \pi^{k(\vec{\mu})} b_{3} & \text { in cases } 2^{\prime}, 4,\end{cases}
$$

where $u_{2,1}(\vec{a}, \lambda)$ is an analytic element bounded by 1 on $\mathcal{H}_{\vec{\mu}} \times S$, defined over the field $\mathbf{Q}_{p}(\pi)$. Notice that in cases $0,1^{\prime}$ (resp. $\left.1^{\prime \prime}, 2^{\prime}, 2^{\prime \prime}, 4\right), u_{2,1}(\vec{a}, \lambda)$ is in fact bounded by $p^{-2}$ (resp. $\left.p^{-1}\right)$.

Estimates for $\gamma_{1,2}$ in all cases. We refer again to formula (3.7), where in any case $\vec{v}-w_{2} \in H^{+}(\mathbf{Z})$, so that only terms $A_{\vec{\mu}+p \vec{v}}(\lambda) \Lambda_{2, \vec{v}}\left(\vec{b}, \lambda^{p}\right)$ with $k(\vec{v}) \geq 1$ appear. Therefore we obtain

$$
\left\|\gamma_{1,2}(\vec{a}, \lambda)\right\|_{\vec{\mu}} \leq\left|p \pi^{k(\vec{\mu})}\right|
$$

The conclusion is that

$$
\gamma_{1,2}(\vec{a}, \lambda)=p \pi^{k(\vec{\mu})} u_{1,2}(\vec{a}, \lambda),
$$

where $u_{1,2}(\vec{a}, \lambda)$ is an analytic element bounded by 1 on $\mathcal{H}_{\vec{\mu}} \times S$, defined over the field $\mathbf{Q}_{p}(\pi)$.

Calculation of $\gamma_{2,2}^{0}$ in cases $0,2,2^{\prime}$ and $2^{\prime \prime}$. We take

$$
\gamma_{2,2}^{0}(\vec{a}, \lambda)=A_{\vec{\mu}-w_{2}+p w_{2}}(\vec{a}, \lambda) .
$$

We check from (3.8) that

$$
|| \gamma_{2,2}(\vec{a}, \lambda)-\gamma_{2,2}^{0}(\vec{a}, \lambda) \|_{\vec{\mu}} \leq\left|p^{2} \pi^{k(\vec{\mu})}\right| .
$$

We explicitly calculate

$$
\begin{aligned}
& \gamma_{2,2}^{0}(a, \lambda)=A_{\vec{\mu}-w_{2}+p w_{2}}(\lambda) \\
& =\frac{\pi^{\mu_{1}+\mu_{2}+p-1}}{\mu_{1} !\left(\mu_{2}+p-1\right) !} \sum_{t=0}^{\min \left(\mu_{1}, \mu_{4}\right)}\left(\begin{array}{c}
\mu_{1} \\
t
\end{array}\right)\left(\begin{array}{c}
\mu_{2}+p-1 \\
\mu_{4}-t
\end{array}\right) \lambda^{t} \\
& =\left((-1)^{\mu_{4}} \gamma_{p}\left(-\mu_{1}, 0\right) \gamma_{p}\left(-\mu_{2}-p,-1\right)\right) \\
& \cdot(-1)^{\mu_{b}}\left(\mu_{b}-\mu_{c}+p\right) \sum_{s+t=\mu_{b}}\left(\begin{array}{c}
\mu_{b}-\mu_{c}+p-1 \\
s
\end{array}\right)\left(\begin{array}{c}
\mu_{a} \\
t
\end{array}\right) \lambda^{t} .
\end{aligned}
$$


We put

$$
P_{2,2}^{\vec{\mu}}(\lambda)=\sum_{s+t=\mu_{b}}\left(\begin{array}{c}
\mu_{b}-\mu_{c}+p-1 \\
s
\end{array}\right)\left(\begin{array}{c}
\mu_{a} \\
t
\end{array}\right) \lambda^{t}
$$

and define again, in cases $2,2^{\prime}$ and $2^{\prime \prime}$, the Hasse domain $S^{\vec{\mu}}$ of type $\vec{\mu}$ to be the region

$$
S^{\vec{\mu}}=\left\{\lambda \in S|| P_{2,2}^{\vec{\mu}}(\lambda) \mid=1\right\} .
$$

We recall that the notation in case 0 conflicts with that of (3.14): see the convention we agreed after that formula. Once again $D \subset S^{\vec{\mu}}$ and $S^{\vec{\mu}}$ is stable under the map $\lambda \longmapsto \lambda^{p}$. The conclusion is that

$$
\gamma_{2,2}(\vec{a}, \lambda)=p \pi^{k(\vec{\mu})} u_{2,2}(\vec{a}, \lambda),
$$

where $u_{2,2}(\vec{a}, \lambda)$ represents an analytic element bounded by 1 on $\mathcal{H}_{\vec{\mu}} \times S$, defined over the field $\mathbf{Q}_{p}(\pi)$, which is of constant absolute value 1 on $\mathcal{H}_{\vec{\mu}} \times S^{\vec{\mu}}$.

Estimates for $\gamma_{2,2}$ in cases $1,1^{\prime}, 1^{\prime \prime}, 3,4$. We refer again to formula (3.7), where $\vec{v}-w_{2} \in H^{+}(\mathbf{Z})$. A calculation similar to the one of (3.23) shows that

$$
\gamma_{2,2}(\vec{a}, \lambda)=p \pi^{k(\vec{\mu})} u_{2,2}(\vec{a}, \lambda),
$$

where $u_{2,2}(a, \lambda)$ represents an analytic element bounded by 1 on $\mathcal{H}_{\vec{\mu}} \times S$, defined over the field $\mathbf{Q}_{p}(\pi)$.

We summarize the results of this section.

Theorem 3.30. Let $p \geq 7$ and $0 \leq \epsilon \leq p^{-1}\left(1-\frac{1}{p}-\frac{1}{p-1}\right)$. Let us fix $\vec{\mu} \in \mathcal{S}$. The matrix $\gamma_{g}\left(\vec{a}, \frac{\vec{a}+\vec{\mu}}{p} ; \lambda\right)$, a priori defined by (2.6.1) when (3.1) is satisfied and $(\vec{a}, \lambda) \in \mathcal{H}_{\vec{\mu}} \times S_{\epsilon}$, extends to a matrix of analytic functions on $\mathcal{H}_{\vec{\mu}} \times S$. We still denote this extension by

$$
\gamma_{g}\left(\vec{a}, \frac{\vec{a}+\vec{\mu}}{p} ; \lambda\right)=\left(\begin{array}{cc}
\gamma_{11} & \gamma_{12} \\
\gamma_{21} & \gamma_{22}
\end{array}\right) .
$$

We have, depending on the case of $\vec{\mu}$ :

Cases 0, 1, 1', $1^{\prime \prime}$.

$$
\gamma_{g}\left(\vec{a}, \frac{\vec{a}+\vec{\mu}}{p} ; \lambda\right)=\pi^{k(\vec{\mu})}\left(\begin{array}{cc}
u_{1,1} & u_{1,2} \\
q \lambda u_{2,1} & u_{2,2}
\end{array}\right)\left(\begin{array}{cc}
\lambda^{\mu_{c}} & 0 \\
0 & p
\end{array}\right) .
$$

Case 2.

$$
\gamma_{g}\left(\vec{a}, \frac{\vec{a}+\vec{\mu}}{p} ; \lambda\right)=p \pi^{k(\vec{\mu})}\left(\begin{array}{cc}
u_{1,1} & u_{1,2} \\
q \lambda u_{2,1} & u_{2,2}
\end{array}\right)\left(\begin{array}{cc}
p \lambda^{\mu_{c}} & 0 \\
0 & 1
\end{array}\right)\left(\begin{array}{cc}
b_{2} b_{3} & 0 \\
0 & 1
\end{array}\right) .
$$

Cases $2^{\prime \prime}, 3$.

$$
\gamma_{g}\left(\vec{a}, \frac{\vec{a}+\vec{\mu}}{p} ; \lambda\right)=p \pi^{k(\vec{\mu})}\left(\begin{array}{cc}
u_{1,1} & u_{1,2} \\
q \lambda u_{2,1} & u_{2,2}
\end{array}\right)\left(\begin{array}{cc}
\lambda^{\mu_{c}} & 0 \\
0 & 1
\end{array}\right)\left(\begin{array}{cc}
b_{2} & 0 \\
0 & 1
\end{array}\right) .
$$


Cases 2', 4.

$$
\gamma_{g}\left(\vec{a}, \frac{\vec{a}+\vec{\mu}}{p} ; \lambda\right)=p \pi^{k(\vec{\mu})}\left(\begin{array}{cc}
u_{1,1} & u_{1,2} \\
q \lambda u_{2,1} & u_{2,2}
\end{array}\right)\left(\begin{array}{cc}
\lambda^{\mu_{c}} & 0 \\
0 & 1
\end{array}\right)\left(\begin{array}{cc}
b_{3} & 0 \\
0 & 1
\end{array}\right) .
$$

Here $q$ is given by the Table 3.30.1.

TABLE 3.30 .1

\begin{tabular}{|c|c|c|c|c|c|c|c|c|c|}
\hline Case & 0 & 1 & $1^{\prime}$ & $1^{\prime \prime}$ & 2 & $2^{\prime}$ & $2^{\prime \prime}$ & 3 & 4 \\
\hline$q$ & $p^{2} b_{2} b_{3}$ & 1 & $p b_{3}$ & $p b_{2}$ & 1 & $p b_{2}$ & $p b_{3}$ & 1 & 1 \\
\hline
\end{tabular}

In all cases the functions $u_{i, j}$, for $i, j=1,2$, are analytic elements on $\mathcal{H}_{\vec{\mu}} \times S$, defined over $\mathbf{Q}_{p}(\pi)$ and bounded by 1 , and

$$
u(\vec{a}, \lambda)=\operatorname{det}\left(\begin{array}{cc}
u_{1,1} & u_{1,2} \\
q \lambda u_{2,1} & u_{2,2}
\end{array}\right)
$$

is an analytic element of constant absolute value 1 on $\mathcal{H}_{\vec{\mu}} \times S$. In cases $0,1,1^{\prime}$, $1^{\prime \prime}$ (resp. in cases $\left.0,2,2^{\prime}, 2^{\prime \prime}\right) u_{1,1}$ (resp. $u_{2,2}$ ) has constant absolute value 1 on $\mathcal{H}_{\vec{\mu}} \times S^{\vec{\mu}}$, where $S^{\vec{\mu}}$ denotes the Hasse domain of type $\vec{\mu}$. (We recall the convention following (3.14) for case 0.)

Proof. Only the assertion concerning $u(\vec{a}, \lambda)$ needs to be proved. We have

$$
\operatorname{det} \gamma_{g}(\vec{a}, \vec{b}, \lambda)=\pi^{2 k(\vec{\mu})} \lambda^{\mu_{c}} u(\vec{a}, \lambda) \begin{cases}p & \text { in cases } 0,1,1^{\prime}, 1^{\prime \prime}, \\ p^{3} b_{2} b_{3} & \text { in case 2, } \\ p^{2} b_{2} & \text { in cases } 2^{\prime \prime}, 3 \\ p^{2} b_{3} & \text { in cases } 2^{\prime}, 4\end{cases}
$$

where

$$
u(\vec{a}, \lambda)=\operatorname{det}\left(\begin{array}{cc}
u_{1,1} & u_{1,2} \\
q \lambda u_{2,1} & u_{2,2}
\end{array}\right)
$$

represents an analytic element defined over the field $\mathbf{Q}_{p}(\pi)$ on $\mathcal{H}_{\vec{\mu}} \times S$. The determinant formula [GHF, 13.6] and the modular properties of the $p$-adic gamma function (1.17) show that $u(\vec{a}, \lambda)$ has absolute value 1 on $\mathcal{H}_{\vec{\mu}} \times S$.

Q.E.D.

Remark 3.32.1. The constant terms of the $u_{i, j}$ 's, i.e. the values $u_{i, j}(0)$, are all units except possibly for $u_{2,1}(0)$ when $\mu_{c}=p-1$ and $u_{1,2}(0)$ when $\mu_{c}=0$. This may be most easily deduced from formulas (3.37.5) and (3.37.7) below, taking into account (3.37.10). Professor Dwork has informed us that in terms of the logarithmic derivative of the Morita $p$-adic gamma function $G=\frac{\Gamma_{p}{ }^{\prime}}{\Gamma_{p}}$ one has the following relevant formulas. For $\mu_{c}=p-1$

$$
u_{2,1}(0) \sim[G(0)-G(a)]+[G(0)-G(b)]+p(1-c) ;
$$

for $\mu_{c}=0$

$$
u_{1,2}(0) \sim[G(0)-G(a)]+[G(0)-G(b)]+p c^{\prime} .
$$


This is also a consequence of formulas (3.37.6) and (3.37.8) below, but requires some analysis of the Taylor expansion of $\Gamma_{p}(z)$.

We now rephrase Lemma 24.4 of [LDE]. We first introduce the functions of $(a, b, c ; A, B, C)$, defined for $\vec{\nu}=\left(\mu_{a, A}, \mu_{b, B}, \mu_{c, C}\right) \in \mathbf{Z}^{3},(a, b, c) \in \mathcal{H}_{\vec{\nu}}^{\prime}$, and $(A, B, C)$ $=p^{-1}((a, b, c)+\vec{\nu})$ :

$$
\begin{gathered}
\xi_{1}^{(0)}(a, b, c ; A, B, C)=\frac{\gamma_{p}(b, B) \gamma_{p}(c-b, C-B)}{\gamma_{p}(1+c, 1+C)} \\
\xi_{2}^{(0)}(a, b, c ; A, B, C)=(-1)^{\mu_{b, B}-\mu_{c, C}} \frac{\gamma_{p}(c-1, C-1) \gamma_{p}(1-a, 1-A)}{\gamma_{p}(1+c-a, 1+C-A)} .
\end{gathered}
$$

Under the same assumptions, formula (2.20) defines the matrix $B(a, b, c ; A, B, C ; \lambda)$, provided $\lambda \in S \backslash\{0\}$ and condition (2.18.2) is satisfied. If $\vec{\nu}=\left(\mu_{a}, \mu_{b}, \mu_{c}\right) \in \mathcal{S}^{\prime}=$ $\{0, \ldots, p-1\}^{3}$, we set in particular

$$
\begin{gathered}
(A, B, C)=\left(a^{\prime}, b^{\prime}, c^{\prime}\right), \\
\xi_{i}^{(0)}\left(a, b, c ; a^{\prime}, b^{\prime}, c^{\prime}\right)=\xi_{i}^{(0)}(a, b, c), \text { for } i=1,2,
\end{gathered}
$$

and

$$
\mathbf{B}\left(a, b, c ; a^{\prime}, b^{\prime}, c^{\prime} ; \lambda\right)=\mathbf{B}(a, b, c ; \lambda) .
$$

Corollary 3.37. Let $p \geq 7, \vec{\mu} \in \mathcal{S}$ and $\vec{\nu}=T_{I}(\vec{\mu})=\left(\mu_{a}, \mu_{b}, \mu_{c}\right) \in \mathcal{S}^{\prime}$. The matrix $\mathbf{B}(a, b, c ; \lambda)$, defined by (2.20) for $(a, b, c ; \lambda) \in \mathcal{H}_{\vec{\nu}}^{\prime} \times(S \backslash\{0\})$ if condition (2.18.2) holds, is related to $\gamma(\vec{a}, \vec{b} ; \lambda)$ by (2.16.1), (2.16.2), (2.16.3) and (2.21), where $\vec{b}=\frac{\vec{a}+\vec{\mu}}{p}$. The matrix $\mathbf{B}(a, b, c ; \lambda)$ extends to a matrix of analytic functions on the region $\mathcal{H}_{\vec{\nu}}^{\prime} \times S$. We have, depending on the case of $\vec{\nu}($ i.e. of $\vec{\mu})$ :

Cases 1, 1'.

$$
\mathbf{B}(a, b, c ; \lambda)=\left(\begin{array}{cc}
1 & 0 \\
0 & p\left(c^{\prime}-b^{\prime}\right)
\end{array}\right)\left(\begin{array}{cc}
\lambda^{\mu_{c}} & 0 \\
0 & 1
\end{array}\right)\left(\begin{array}{cc}
\beta_{1} & q \lambda \beta_{2} \\
q^{\prime} \beta_{3} & \beta_{4}
\end{array}\right) .
$$

Case 2, 2'.

$$
\mathbf{B}(a, b, c ; \lambda)=\left(\begin{array}{cc}
p\left(a^{\prime}-c^{\prime}\right) & 0 \\
0 & 1
\end{array}\right)\left(\begin{array}{cc}
\lambda^{\mu_{c}} & 0 \\
0 & 1
\end{array}\right)\left(\begin{array}{cc}
\beta_{1} & q \lambda \beta_{2} \\
q^{\prime} \beta_{3} & \beta_{4}
\end{array}\right) .
$$

Case 0, 1", 2", 3 .

$$
\mathbf{B}(a, b, c ; \lambda)=\left(\begin{array}{cc}
\lambda^{\mu_{c}} & 0 \\
0 & 1
\end{array}\right)\left(\begin{array}{cc}
\beta_{1} & q \lambda \beta_{2} \\
q^{\prime} \beta_{3} & \beta_{4}
\end{array}\right) .
$$

Case 4.

$$
\mathbf{B}(a, b, c ; \lambda)=\left(\begin{array}{cc}
p\left(a^{\prime}-c^{\prime}\right) & 0 \\
0 & p\left(c^{\prime}-b^{\prime}\right)
\end{array}\right) \cdot\left(\begin{array}{cc}
\lambda^{\mu_{c}} & 0 \\
0 & 1
\end{array}\right)\left(\begin{array}{cc}
\beta_{1} & \lambda \beta_{2} \\
\beta_{3} & \beta_{4}
\end{array}\right)
$$

where $q, q^{\prime}$ are given by the Table 3.37.1. 
TABLE 3.37 .1

\begin{tabular}{|c|c|c|c|c|c|c|c|c|c|}
\hline Case & 0 & 1 & $1^{\prime}$ & $1^{\prime \prime}$ & 2 & $2^{\prime}$ & $2^{\prime \prime}$ & 3 & 4 \\
\hline$q$ & $p\left(a^{\prime}-c^{\prime}\right)$ & 1 & $p\left(a^{\prime}-c^{\prime}\right)$ & 1 & 1 & 1 & $p\left(a^{\prime}-c^{\prime}\right)$ & 1 & 1 \\
\hline$q^{\prime}$ & $p\left(b^{\prime}-c^{\prime}\right)$ & 1 & 1 & $p\left(b^{\prime}-c^{\prime}\right)$ & 1 & 1 & 1 & 1 & 1 \\
\hline
\end{tabular}

In all cases the functions $\beta_{i}$, for $i=1,2,3,4$, are analytic elements defined over $\mathbf{Q}_{p}(\pi)$ and bounded by 1 on $\mathcal{H}_{\vec{\nu}} \times S$. The analytic element on $\mathcal{H}_{\vec{\nu}}^{\prime} \times S$

$$
\beta(a, b, c ; \lambda)=\operatorname{det}\left(\begin{array}{cc}
\beta_{1} & q \lambda \beta_{2} \\
q^{\prime} \beta_{3} & \beta_{4}
\end{array}\right)
$$

has constant absolute value 1 . In cases $0,1,1^{\prime}, 1^{\prime \prime}$ (resp. 0, 2, 2', 2") $\beta_{1}$ (resp. $\beta_{4}$ ) has constant absolute value 1 on $\mathcal{H}_{\vec{\nu}}^{\prime} \times S^{\vec{\nu}}$, where $S^{\vec{\nu}}=S^{\vec{\mu}}$ denotes the Hasse domain of type $\vec{\mu}$. (We recall the convention following (3.14) for case 0.) At $\lambda=0$, we have, as meromorphic functions on $\mathcal{H}_{\vec{\nu}}^{\prime}$ :

$$
\begin{gathered}
\left(\lambda^{-\mu_{c}} \mathbf{B}_{1}(a, b, c ; \lambda)\right)_{\lambda=0}=\frac{1-c}{1-c^{\prime}} \xi_{2}^{(0)}(a, b, c) \\
\mathbf{B}_{4}(a, b, c ; 0)=\frac{c}{c^{\prime}} \xi_{1}^{(0)}(a, b, c)
\end{gathered}
$$

If $\mu_{c}<p-1$, we have

$$
\left(\lambda^{-\mu_{c}-1} \mathbf{B}_{2}(a, b, c ; \lambda)\right)_{\lambda=0}=\frac{c-a}{1-c^{\prime}} \xi_{2}^{(0)}(a, b, c),
$$

a p-adic unit. If $\mu_{c}=p-1$, we have

$$
\left(\lambda^{-p} \mathbf{B}_{2}(a, b, c ; \lambda)\right)_{\lambda=0}=\frac{c-a}{1-c^{\prime}} \xi_{2}^{(0)}(a, b, c)-\frac{c}{c^{\prime}} \frac{c^{\prime}-a^{\prime}}{1-c^{\prime}} \xi_{1}^{(0)}(a, b, c)
$$

If $\mu_{c}>0$, we have

$$
\mathbf{B}_{3}(a, b, c ; 0)=\frac{c-b}{c^{\prime}} \xi_{1}^{(0)}(a, b, c)
$$

a p-adic unit. If $\mu_{c}=0$, we have

$$
\mathbf{B}_{3}(a, b, c ; 0)=\frac{c-b}{c^{\prime}} \xi_{1}^{(0)}(a, b, c)-\frac{1-c}{c^{\prime}} \frac{c^{\prime}-b^{\prime}}{1-c^{\prime}} \xi_{2}^{(0)}(a, b, c) .
$$

N.B. 3.37.9. One may easily verify by direct calculation that the functions in $(3.37 .3,4,5,6,7,8)$ are analytic in $\mathcal{H}_{\vec{\nu}}^{\prime}$, under the indicated assumptions.

Proof. We rewrite formula (2.21):

$$
\begin{aligned}
\left(\begin{array}{ll}
\mathbf{B}_{1} & \mathbf{B}_{2} \\
\mathbf{B}_{3} & \mathbf{B}_{4}
\end{array}\right)= & (-1)^{\mu_{4}} \gamma_{p}\left(a_{1}, b_{1}\right)^{-1} \gamma_{p}\left(a_{2}, b_{2}\right)^{-1} \\
& \cdot\left(\begin{array}{cc}
1 & 0 \\
0 & -b_{2}
\end{array}\right)\left(\begin{array}{cc}
\gamma_{11} & \gamma_{21} \\
\gamma_{12} & \gamma_{22}
\end{array}\right)\left(\begin{array}{cc}
1 & 0 \\
0 & -\frac{1}{a_{2}}
\end{array}\right) .
\end{aligned}
$$


We recall that $a_{1}=a, a_{2}=b-c, a_{3}=a-c, a_{4}=b$. The result, except for formulas (3.37.4) and (3.37.6), follows from simultaneous consideration of the theorem and [LDE, Lemma 24.4 and Chapter 25]. The statement about $\mathbf{B}_{2}(a, b, c ; \lambda)$ (resp. $\left.\mathbf{B}_{2}(a, b, c ; \lambda)\right)$ in [LDE, Lemma 24.4] is in error (resp. is incomplete) when $\mu_{c}=p-1$ (resp. $\left.\mu_{c}=0\right)$. The correct statement is (3.37.6) (resp. (3.37.8)). To prove (3.37.6) one follows the proof of [LDE, Lemma 24.4], but needs to calculate (in the notation of loc. cit.)

$$
V\left(\lambda^{p}\right)^{-1} \equiv-\frac{1}{c^{\prime}\left(1-c^{\prime}\right)}\left(\begin{array}{cc}
0 & -c^{\prime} \\
-\left(1-c^{\prime}\right) & c^{\prime}-b^{\prime}
\end{array}\right)+\lambda^{p}\left(\begin{array}{cc}
\frac{c^{\prime}-a^{\prime}}{c^{\prime}\left(c^{\prime}-1\right)} & * \\
* & *
\end{array}\right) \bmod \lambda^{2 p},
$$

where $*$ denotes an unspecified constant. The conclusion follows as in loc. cit. As for (3.37.8), one simply needs to carry on one step the calculation in loc. cit. Q.E.D.

N.B. 3.37.11. Our corollary extends the results of [Dw1, §4] into the singular disk at $\lambda=0$.

\section{Bounded SOLutions $(p \geq 7)$}

For $a=a^{(0)} \in \mathbf{Z}_{p}$, we define $a^{\prime}=a^{(1)} \in \mathbf{Z}_{p}$ and $\mu_{a} \in\{0, \ldots, p-1\}$ by the condition that $\mu_{a}=p a^{\prime}-a$. For $j=2,3, \ldots$, we then put $a^{(j)}=\left(a^{(j-1)}\right)^{\prime}$. Therefore

$$
-a=\mu_{a}+\mu_{a^{\prime}} p+\cdots+\mu_{a^{(j)}} p^{j}+\cdots
$$

is the Hensel expansion of $-a \in \mathbf{Z}_{p}$. We now consider the subset $\mathcal{T}_{1}$ (resp. $\mathcal{T}_{2}$ ) of $\mathbf{Z}_{p}^{3}$ consisting of triples $(a, b, c)$ such that for all $j=0,1,2, \ldots,\left(\mu_{a^{(j)}}, \mu_{b^{(j)}}, \mu_{c^{(j)}}\right)$ is in case 1 or $1^{\prime}$ (resp. in case 2 or $2^{\prime}$ ) of the previous section. We define the strict Hasse domain of case $i$, for $i=1,2$, as

$$
S_{i}^{H}=\bigcap S^{\vec{\mu}},
$$

where the intersection is taken for all $\vec{\mu}$ in case $i$ or $i^{\prime}$. Notice that, for $i=1,2, S_{i}^{H}$ is the complement in $S$ of a finite number of open disks of radius 1 , and $D \subset S_{i}^{H}$. We also define, for $i=1,2$,

$$
\mathcal{H}_{i}^{\prime}=\bigcup \mathcal{H}_{\vec{\nu}}^{\prime}
$$

where the union is taken for all $\vec{\nu}$ in case $i$.

Definition 4.3.0. We say that $c \in \mathbf{Z}_{p}$ is strongly of type 1 or anti-Liouville if $\mu_{c^{(j)}} \geq 1$ for all $j \in \mathbf{N}$.

An anti-Liouville number is of type 1 in the sense of [B-C]. If $c$ is anti-Liouville, then $c$ is a limit of rational, non-integral, anti-Liouville numbers. In fact, if $-c=$ $\sum_{i=0}^{i=\infty} \mu_{i} p^{i}$, let $-\gamma_{s}=\sum_{i=0}^{s-1} \mu_{i} p^{i}+p^{s}\left(1+2 p+p^{2}+2 p^{3}+\ldots\right)$, which is non-integral since $\frac{1+2 p}{p^{2}-1} \notin \mathbf{Z}$; then $\lim _{s} \gamma_{s}=c$. Clearly, $1-c$ is anti-Liouville iff $\mu_{c^{(j)}} \leq p-2$ for all $j \in \mathbf{N}$. No element of $\mathbf{Z}_{\leq 0}$ is anti-Liouville. If $(a, b, c) \in \mathcal{T}_{1}$ (resp. $\mathcal{T}_{2}$ ) then $1-c$ (resp. $c$ ) is anti-Liouville. In particular, if $(a, b, c) \in \mathcal{T}_{1}$ (resp. $\mathcal{T}_{2}$ ), then $c \notin \mathbf{Z}_{>0}$ 
(resp. $c \notin \mathbf{Z}_{\leq 0}$ ). If $1-c$ (resp. $c$ ) is anti-Liouville, so in particular if $(a, b, c) \in \mathcal{T}_{1}$ (resp. $\mathcal{T}_{2}$ ), then

$$
|c-s|>\frac{|p|}{s}, \text { for } s \in \mathbf{Z}_{>0}
$$

(resp.

$$
\left.|c+s|>\frac{|p|}{s}, \text { for } s \in \mathbf{Z}_{>0}\right) .
$$

Notice that the transformation

$$
(a, b, c) \longmapsto(1-b, 1-a, 1-c)
$$

induces a bijection

$$
\mathcal{T}_{1} \longrightarrow \mathcal{T}_{2}
$$

Let $K$ denote any finite extension of $\mathbf{Q}_{p}(\pi)$, and let $\sigma$ be a Frobenius automorphism of $K$, such that $\sigma(\pi)=\pi$. Let $T$ denote any admissible open subset of the $K$ rigid analytic projective line $X$. We may interpret $T$ as an admissible open subset "defined over $K$ " of the extension of $X$ to $\mathbf{C}_{p}$.

Definition 4.5. Let $T$ be as before and $\mathcal{T}$ a subset of $\left(\mathbf{Z}_{p}\right)^{3}$. Define $\mathcal{B}_{K}(\mathcal{T} \times T)$ to be the $K$-Banach algebra of continuous bounded $\mathbf{C}_{p}$-valued functions $f=$ $f(a, b, c ; \lambda)$ on $\mathcal{T} \times T$, such that for each choice of $(a, b, c)$ in $\mathcal{T}, \lambda \longmapsto f(a, b, c ; \lambda)$ is an analytic function on $T$, defined over $K$. We denote by $\|-\| \mathcal{T} \times T$ or simply by $\|-\|$ the norm of $\mathcal{B}_{K}(\mathcal{T} \times T)$, i.e. the supnorm on $\mathcal{T} \times T$, and by $\mathcal{B}_{K}^{(1)}(\mathcal{T} \times T)$ the unit ball of $\mathcal{B}_{K}(\mathcal{T} \times T)$.

If $0 \in T$ and $T$ is bounded, the principal ideal $\lambda \mathcal{B}_{K}(\mathcal{T} \times T)\left(\right.$ resp. $\left.\lambda \mathcal{B}_{K}^{(1)}(\mathcal{T} \times T)\right)$ generated by the bounded analytic function (projection onto the last factor) $\lambda$ : $(a, b, c ; \lambda) \longmapsto \lambda$ is closed and is the kernel of the map:

$$
\begin{aligned}
\mathcal{B}_{K}(\mathcal{T} \times T) & \longrightarrow \mathcal{C}_{K}(\mathcal{T}), \\
f(a, b, c ; \lambda) & \longmapsto f(a, b, c ; 0)
\end{aligned}
$$

(resp.

$$
\begin{aligned}
\mathcal{B}_{K}^{(1)}(\mathcal{T} \times T) & \longrightarrow \mathcal{C}_{K}^{(1)}(\mathcal{T}), \\
f(a, b, c ; \lambda) & \longmapsto f(a, b, c ; 0)),
\end{aligned}
$$

where $\mathcal{C}_{K}(\mathcal{T})\left(\right.$ resp. $\left.\mathcal{C}_{K}^{(1)}(\mathcal{T})\right)$ denotes the $K$-Banach algebra of continuous $K$-valued functions on $\mathcal{T} i$ (resp. its unit ball). Let us assume that $T^{\sigma}=T$ and that $T$ is stable under the map $\lambda \longmapsto \lambda^{p}$. For $i=1,2$, we consider the map:

$$
\begin{aligned}
\phi: \mathcal{T}_{i} \times T & \longrightarrow \mathcal{T}_{i} \times T, \\
(a, b, c, \lambda) & \longmapsto\left(a^{\prime}, b^{\prime}, c^{\prime}, \lambda^{p}\right) .
\end{aligned}
$$


For a $\mathbf{C}_{p}$-valued function $w \in \mathcal{B}_{K}\left(\mathcal{T}_{i} \times T\right)$ we then denote by $w^{\sigma \phi}$ (resp. $w^{\sigma}$ ) the composition $\bar{\sigma} \circ w \circ \phi \circ \bar{\sigma}^{-1}$ (resp. $\bar{\sigma} \circ w \circ \bar{\sigma}^{-1}$ ), where $\bar{\sigma}$ denotes any isometric extension of $\sigma$ to $\mathbf{C}_{p}$. Then $w \longmapsto w^{\sigma \phi}$ (resp. $w \longmapsto w^{\sigma}$ ) induces an isometry of $\mathcal{B}_{K}\left(\mathcal{T}_{i} \times T\right)$. The previous assumptions are clearly satisfied by $T=D$ and by $T=S_{i}^{H}$, for $i=1,2$.

We now distinguish the case $(a, b, c) \in \mathcal{T}_{1}$ from the case $(a, b, c) \in \mathcal{T}_{2}$.

Case 1. There exists a map

$$
C_{1}: \lambda \mathcal{B}_{K}^{(1)}\left(\mathcal{T}_{1} \times D\right) \longrightarrow \lambda \mathcal{B}_{K}^{(1)}\left(\mathcal{T}_{1} \times D\right)
$$

that induces

$$
C_{1}: \lambda \mathcal{B}_{K}^{(1)}\left(\mathcal{T}_{1} \times S_{1}^{H}\right) \longrightarrow \lambda \mathcal{B}_{K}^{(1)}\left(\mathcal{T}_{1} \times S_{1}^{H}\right)
$$

defined by

$$
\begin{aligned}
C_{1}(\lambda w) & =\frac{\mathbf{B}_{2}+\mathbf{B}_{4} \lambda^{p} w^{\sigma \phi}}{\mathbf{B}_{1}+\mathbf{B}_{3} \lambda^{p} w^{\sigma \phi}}=\frac{\lambda^{\mu_{c}+1} \beta_{2}+p\left(c^{\prime}-b^{\prime}\right) \beta_{4} \lambda^{p} w^{\sigma \phi}}{\lambda^{\mu_{c}} \beta_{1}+p\left(c^{\prime}-b^{\prime}\right) \beta_{3} \lambda^{p} w^{\sigma \phi}} \\
& =\lambda \frac{\beta_{2} \beta_{1}^{-1}+p\left(c^{\prime}-b^{\prime}\right) \beta_{4} \beta_{1}^{-1} \lambda^{p-1-\mu_{c}} w^{\sigma \phi}}{1+p\left(c^{\prime}-b^{\prime}\right) \beta_{3} \beta_{1}^{-1} \lambda^{p-\mu_{c}} w^{\sigma \phi}} .
\end{aligned}
$$

We observe that, for $w_{1}, w_{2} \in \lambda \mathcal{B}_{K}^{(1)}\left(\mathcal{T}_{1} \times D\right)\left(\right.$ resp. $\left.\lambda \mathcal{B}_{K}^{(1)}\left(\mathcal{T}_{1} \times S_{1}^{H}\right)\right)$,

$$
\begin{gathered}
C_{1}\left(\lambda w_{1}\right)-C_{1}\left(\lambda w_{2}\right)=\frac{\lambda^{p} \operatorname{det} \mathbf{B}\left(w_{1}^{\sigma \phi}-w_{2}^{\sigma \phi}\right)}{\left(\mathbf{B}_{1}+\mathbf{B}_{3} \lambda^{p} w_{1}^{\sigma \phi}\right)\left(\mathbf{B}_{1}+\mathbf{B}_{3} \lambda^{p} w_{2}^{\sigma \phi}\right)} \\
=\frac{p\left(c^{\prime}-b^{\prime}\right) \lambda^{p+\mu_{c}} \beta(a, b, c ; \lambda)\left(w_{1}^{\sigma \phi}-w_{2}^{\sigma \phi}\right)}{\left(\lambda^{\mu_{c}} \beta_{1}+p\left(c^{\prime}-b^{\prime}\right) \beta_{3} \lambda^{p} w_{1}^{\sigma \phi}\right)\left(\lambda^{\mu_{c}} \beta_{1}+p\left(c^{\prime}-b^{\prime}\right) \beta_{3} \lambda^{p} w_{2}^{\sigma \phi}\right)} \\
=p\left(c^{\prime}-b^{\prime}\right) \lambda^{p-\mu_{c}} \beta_{1}^{-2} \beta(a, b, c ; \lambda) \\
\cdot \frac{\left(w_{1}^{\sigma \phi}-w_{2}^{\sigma \phi}\right)}{\left(1+p\left(c^{\prime}-b^{\prime}\right) \beta_{3} \beta_{1}^{-1} \lambda^{p-\mu_{c}} w_{1}^{\sigma \phi}\right)\left(1+p\left(c^{\prime}-b^{\prime}\right) \beta_{3} \beta_{1}^{-1} \lambda^{p-\mu_{c}} w_{2}^{\sigma \phi}\right)}
\end{gathered}
$$

We conclude that

$$
\begin{aligned}
\left\|\frac{C_{1}\left(\lambda w_{1}\right)}{\lambda}-\frac{C_{1}\left(\lambda w_{2}\right)}{\lambda}\right\| & \leq\left\|p\left(c^{\prime}-b^{\prime}\right) \lambda^{p-\mu_{c}-1} \beta_{1}^{-2} \beta\right\|\left\|w_{1}-w_{2}\right\| \\
& \leq p^{-1}\left\|w_{1}-w_{2}\right\| .
\end{aligned}
$$

The map $w \longmapsto \frac{C_{1}(\lambda w)}{\lambda}$ is then a contraction of the complete metric space $\mathcal{B}_{K}^{(1)}\left(\mathcal{T}_{1} \times D\right)\left(\right.$ resp. $\left.\hat{\mathcal{B}}_{K}^{(1)}\left(\mathcal{T}_{1} \times S_{1}^{H}\right)\right)$ and as such it admits a unique fixed point. The map $C_{1}$ therefore also admits a unique fixed point

$$
\eta(a, b, c ; \lambda) \in \lambda \mathcal{B}_{K}^{(1)}\left(\mathcal{T}_{1} \times S_{1}^{H}\right) \subset \lambda \mathcal{B}_{K}^{(1)}\left(\mathcal{T}_{1} \times S_{1}^{H}\right) .
$$


We then have on $\mathcal{T}_{1} \times S_{1}^{H}$

$$
\eta=\frac{\mathbf{B}_{2}+\mathbf{B}_{4} \eta^{\sigma \phi}}{\mathbf{B}_{1}+\mathbf{B}_{3} \eta^{\sigma \phi}}
$$

Case 2. We now consider the map

$$
C_{2}: \mathcal{B}_{K}^{(1)}\left(\mathcal{T}_{2} \times D\right) \longrightarrow \mathcal{B}_{K}^{(1)}\left(\mathcal{T}_{2} \times D\right)
$$

that induces

$$
C_{2}: \mathcal{B}_{K}^{(1)}\left(\mathcal{T}_{2} \times S_{2}^{H}\right) \longrightarrow \mathcal{B}_{K}^{(1)}\left(\mathcal{T}_{2} \times S_{2}^{H}\right)
$$

and is defined by

$$
\begin{aligned}
C_{2}(w) & =\frac{\mathbf{B}_{3}+\mathbf{B}_{1} w^{\sigma \phi}}{\mathbf{B}_{4}+\mathbf{B}_{2} w^{\sigma \phi}}=\frac{\beta_{3}+p\left(a^{\prime}-c^{\prime}\right) \lambda^{\mu_{c}} \beta_{1} w^{\sigma \phi}}{\beta_{4}+p\left(a^{\prime}-c^{\prime}\right) \lambda^{\mu_{c}+1} \beta_{2} w^{\sigma \phi}} \\
& =\frac{\beta_{3} \beta_{4}^{-1}+p\left(a^{\prime}-c^{\prime}\right) \lambda^{\mu_{c}} \beta_{1} \beta_{4}^{-1} w^{\sigma \phi}}{1+p\left(a^{\prime}-c^{\prime}\right) \lambda^{\mu_{c}+1} \beta_{2} \beta_{4}^{-1} w^{\sigma \phi}} .
\end{aligned}
$$

We observe that, for $w_{1}, w_{2} \in \mathcal{B}_{K}^{(1)}\left(\mathcal{T}_{2} \times D\right)$,

$$
\begin{gathered}
C_{2}\left(w_{1}\right)-C_{2}\left(w_{2}\right)=\frac{\operatorname{det} \mathbf{B}\left(w_{1}^{\sigma \phi}-w_{2}^{\sigma \phi}\right)}{\left(\mathbf{B}_{4}+\mathbf{B}_{2} w_{1}^{\sigma \phi}\right)\left(\mathbf{B}_{4}+\mathbf{B}_{2} w_{2}^{\sigma \phi}\right)} \\
=\frac{p\left(a^{\prime}-c^{\prime}\right) \lambda^{\mu_{c}} \beta(a, b, c ; \lambda)\left(w_{1}^{\sigma \phi}-w_{2}^{\sigma \phi}\right)}{\left(\beta_{4}+p\left(a^{\prime}-c^{\prime}\right) \lambda^{\mu_{c}+1} \beta_{2} w_{1}^{\sigma \phi}\right)\left(\beta_{4}+p\left(a^{\prime}-c^{\prime}\right) \lambda^{\mu_{c}+1} \beta_{2} w_{2}^{\sigma \phi}\right)} \\
=p\left(a^{\prime}-c^{\prime}\right) \lambda^{\mu_{c}} \beta_{4}^{-2} \beta(a, b, c ; \lambda) \\
\cdot \frac{\left(w_{1}^{\sigma \phi}-w_{2}^{\sigma \phi}\right)}{\left(1+p\left(a^{\prime}-c^{\prime}\right) \lambda^{\mu_{c}+1} \beta_{2} \beta_{4}^{-1} w_{1}^{\sigma \phi}\right)\left(1+p\left(a^{\prime}-c^{\prime}\right) \lambda^{\mu_{c}+1} \beta_{2} \beta_{4}^{-1} w_{2}^{\sigma \phi}\right) .}
\end{gathered}
$$

We conclude that

$$
\begin{aligned}
\left\|C_{2}\left(w_{1}\right)-C_{2}\left(w_{2}\right)\right\| & \leq\left\|p\left(a^{\prime}-c^{\prime}\right) \lambda^{\mu_{c}} \beta_{4}^{-2} \beta\right\|\left\|w_{1}-w_{2}\right\| \\
& \leq p^{-1}\left\|w_{1}-w_{2}\right\| .
\end{aligned}
$$

The map $C_{2}$ is then a contraction of the complete metric space $\mathcal{B}_{K}^{(1)}\left(\mathcal{T}_{2} \times D\right)$ (resp. $\left.\mathcal{B}_{K}^{(1)}\left(\mathcal{T}_{2} \times S_{2}^{H}\right)\right)$ and as such it admits a unique fixed point

$$
\eta(a, b, c ; \lambda) \in \mathcal{B}_{K}^{(1)}\left(\mathcal{T}_{2} \times S_{2}^{H}\right) .
$$

We then have on $\mathcal{T}_{2} \times S_{2}^{H}$

$$
\eta=\frac{\mathbf{B}_{3}+\mathbf{B}_{1} \eta^{\sigma \phi}}{\mathbf{B}_{4}+\mathbf{B}_{2} \eta^{\sigma \phi}}
$$


We define, for any $(a, b, c) \in\left(\mathbf{C}_{p}\right)^{3}$ for which it makes sense, the standard Gauss hypergeometric series

$$
F(a, b, c ; \lambda)=\sum_{s=0}^{+\infty} \frac{(a)_{s}(b)_{s}}{(c)_{s} s !} \lambda^{s} \in \mathbf{C}_{p}[[\lambda]],
$$

where, as usual, $(a)_{s}=a(a+1) \ldots(a+s-1)$, and a matrix of power series in $\mathbf{C}_{p}[[\lambda]]$

$$
U^{(0)}(a, b, c ; \lambda)=\left(\begin{array}{ll}
u_{1}^{(0)} & u_{2}^{(0)} \\
u_{3}^{(0)} & u_{4}^{(0)}
\end{array}\right)
$$

$$
=\left(\begin{array}{cc}
(c-b) F(a, b, c+1 ; \lambda) & c F(a, b, c ; \lambda) \\
(1-c) F(b-c, a-c, 1-c ; \lambda) & (c-a) \lambda F(1+b-c, 1+a-c, 2-c ; \lambda)
\end{array}\right) .
$$

We observe that the first (resp. second) line of $U^{(0)}(a, b, c ; \lambda)$ is formally defined when $c \notin \mathbf{Z}_{\leq 0}$ (resp. $c \notin \mathbf{Z}_{>0}$ ). We recall that, whenever defined, a line of the matrix

$$
\left(\begin{array}{cc}
1 & 0 \\
0 & \lambda^{-c}
\end{array}\right) U^{(0)}(a, b, c ; \lambda)
$$

formally represents a solution at 0 of the system

$$
\frac{d}{d \lambda} Y=Y\left(\begin{array}{cc}
-\frac{c}{\lambda} & \frac{c-a}{1-\lambda} \\
\frac{c-b}{\lambda} & \frac{a+b-c}{1-\lambda}
\end{array}\right)
$$

In particular, if $(a, b, c) \in \mathcal{T}_{2}$, then $\left(u_{1}^{(0)}, u_{2}^{(0)}\right)$ is a power-series solution of the system (4.18), while if $(a, b, c) \in \mathcal{T}_{1}$, then $\left(u_{3}^{(0)}, u_{4}^{(0)}\right)$ is a power-series solution of

$$
\frac{d}{d \lambda} Z=Z\left(\begin{array}{cc}
0 & \frac{c-a}{1-\lambda}+\frac{c}{\lambda} \\
\frac{2 c-b}{\lambda} & \frac{a+b-c}{1-\lambda}+\frac{c}{\lambda}
\end{array}\right)
$$

Let us recall the formula [LDE, 21.2.4] (but notice an error in that reference: the factor $(a-1)$ is missing there)

$$
\frac{s !}{(a)_{s}}=(a-1) \sum_{i+j=s}(-1)^{j}\left(\begin{array}{l}
s \\
j
\end{array}\right) \frac{1}{a+j-1} .
$$

Together with the bound (4.3.1) (resp. (4.3.2)) it guarantees that

$$
\max \left(\left|\frac{(b-c)_{s}(a-c)_{s}}{s !(1-c)_{s}}\right|,\left|\frac{(1+b-c)_{s}(1+a-c)_{s}}{s !(2-c)_{s}}\right|\right)<p s
$$

if $1-c$ is anti-Liouville and in particular for $(a, b, c) \in \mathcal{T}_{1}$ (resp.

$$
\min \left(\left|\frac{(a)_{s}(b)_{s}}{s !(c)_{s}}\right|,\left|\frac{(a)_{s}(b)_{s}}{s !(c+1)_{s}}\right|\right)<p s
$$

if $c$ is anti-Liouville and in particular for $\left.(a, b, c) \in \mathcal{T}_{2}\right)$. We conclude that $u_{3}^{(0)}, u_{4}^{(0)}$ (resp. $u_{1}^{(0)}, u_{2}^{(0)}$ ) are elements of

$$
\mathcal{B}_{K}^{(1)}\left(\mathcal{T}_{1} \times D\left(0,\left(p^{-1}\right)^{+}\right)\right) \quad\left(\text { resp. } \mathcal{B}_{K}^{(1)}\left(\mathcal{T}_{2} \times D\left(0,\left(p^{-1}\right)^{+}\right)\right)\right) .
$$

We point out $(c f .(3.33,34))$ that $\xi_{1}^{(0)}(a, b, c)\left(\right.$ resp. $\left.\xi_{2}^{(0)}(a, b, c)\right)$ is an analytic function defined over $K$ of constant absolute value 1 on $\mathcal{H}_{2}^{\prime}$ (resp. $\mathcal{H}_{1}^{\prime}$ ), hence an invertible element of $\mathcal{B}_{K}^{(1)}\left(\mathcal{T}_{2} \times D\left(0,1^{+}\right)\right)\left(\right.$resp. $\mathcal{B}_{K}^{(1)}\left(\mathcal{T}_{1} \times D\left(0,1^{+}\right)\right)$. 
Lemma 4.21. We have the identities

$$
\begin{gathered}
\left(u_{3}^{(0)}, u_{4}^{(0)}\right)^{\sigma \phi} \mathbf{B}=\xi_{2}^{(0)} \lambda^{\mu_{c}}\left(u_{3}^{(0)}, u_{4}^{(0)}\right) \text { in } \mathcal{B}_{K}^{(1)}\left(\mathcal{T}_{1} \times D\left(0,\left(p^{-1}\right)^{+}\right)\right)^{2}, \\
\left(u_{1}^{(0)}, u_{2}^{(0)}\right)^{\sigma \phi} \mathbf{B}=\xi_{1}^{(0)}\left(u_{1}^{(0)}, u_{2}^{(0)}\right) \text { in } \mathcal{B}_{K}^{(1)}\left(\mathcal{T}_{2} \times D\left(0,\left(p^{-1}\right)^{+}\right)\right)^{2} .
\end{gathered}
$$

Proof. We restrict our attention to (4.21.2). Let $(\alpha, \beta, \gamma)$ lie in $\left(\mathbf{Z}_{p} \cap \mathbf{Q}\right)^{3}$ with $\gamma$ non-integral. Then $\gamma^{\prime}$ is also non-integral and, by Lemma 24.3 and Chapter 25 of [LDE],

$$
\begin{aligned}
\left(u_{1}^{(0)}, u_{2}^{(0)}\right)\left(\alpha^{\prime}, \beta^{\prime}, \gamma^{\prime} ; \lambda^{p}\right) & \mathbf{B}\left(\alpha, \beta, \gamma ; \alpha^{\prime}, \beta^{\prime}, \gamma^{\prime} ; \lambda\right) \\
& =\xi_{1}^{(0)}\left(\alpha, \beta, \gamma ; \alpha^{\prime}, \beta^{\prime}, \gamma^{\prime}\right)\left(u_{1}^{(0)}, u_{2}^{(0)}\right)(\alpha, \beta, \gamma ; \lambda),
\end{aligned}
$$

valid for all $\lambda \in D$, so in particular for $\left.\lambda \in D\left(0,\left(p^{-1}\right)^{+}\right)\right)$.

Now let $(a, b, c) \in \mathbf{Z}_{p}^{3}$, with $c$ anti-Liouville. There exists a sequence of elements $(\alpha, \beta, \gamma) \in\left(\mathbf{Z}_{p} \cap \mathbf{Q}\right)^{3}$ with $\gamma$ non-integral and anti-Liouville such that $(\alpha, \beta, \gamma) \longrightarrow$ $(a, b, c)$, and hence $\left(\alpha^{\prime}, \beta^{\prime}, \gamma^{\prime}\right) \longrightarrow\left(a^{\prime}, b^{\prime}, c^{\prime}\right)$. By taking limits we obtain

$$
\begin{aligned}
\left(u_{1}^{(0)}, u_{2}^{(0)}\right)\left(a^{\prime}, b^{\prime}, c^{\prime} ; \lambda^{p}\right) & \mathbf{B}\left(a, b, c ; a^{\prime}, b^{\prime}, c^{\prime} ; \lambda\right) \\
& =\xi_{1}^{(0)}\left(a, b, c ; a^{\prime}, b^{\prime}, c^{\prime}\right)\left(u_{1}^{(0)}, u_{2}^{(0)}\right)(a, b, c ; \lambda),
\end{aligned}
$$

valid for $\left.\lambda \in D\left(0,\left(p^{-1}\right)^{+}\right)\right)$, the point being that the series $\left(u_{1}^{(0)}, u_{2}^{(0)}\right)(\alpha, \beta, \gamma ; \lambda)$ converges termwise to $\left(u_{1}^{(0)}, u_{2}^{(0)}\right)(a, b, c ; \lambda)$, and, since $\gamma$ is anti-Liouville, we may use the universal estimate (4.20.3) to conclude that

$$
\begin{gathered}
\left(u_{1}^{(0)}, u_{2}^{(0)}\right)(\alpha, \beta, \gamma ; \lambda) \longrightarrow\left(u_{1}^{(0)}, u_{2}^{(0)}\right)\left(a, b, c ; \lambda^{p}\right), \\
\left(u_{1}^{(0)}, u_{2}^{(0)}\right)\left(\alpha^{\prime}, \beta^{\prime}, \gamma^{\prime} ; \lambda\right) \longrightarrow\left(u_{1}^{(0)}, u_{2}^{(0)}\right)\left(a^{\prime}, b^{\prime}, c^{\prime} ; \lambda^{p}\right),
\end{gathered}
$$

uniformly on $D\left(0, \rho^{+}\right)$, for each $\rho<1$. In particular this is valid for $\rho=\frac{1}{p}$, and here the series are bounded by 1 . The proof of (4.21.1) is the same except that now $1-\gamma$ and $1-c$ are anti-Liouville.

Q.E.D.

Corollary 4.22. We have the identities

$$
\begin{gathered}
\left(u_{3}^{(0)}, u_{4}^{(0)}\right)^{\sigma \phi} \mathbf{B}=\xi_{2}^{(0)} \lambda^{\mu_{c}}\left(u_{3}^{(0)}, u_{4}^{(0)}\right) \text { in } \mathcal{B}_{K}^{(1)}\left(\mathcal{T}_{1} \times D\right)^{2}, \\
\left(u_{1}^{(0)}, u_{2}^{(0)}\right)^{\sigma \phi} \mathbf{B}=\xi_{1}^{(0)}\left(u_{1}^{(0)}, u_{2}^{(0)}\right) \text { in } \mathcal{B}_{K}^{(1)}\left(\mathcal{T}_{2} \times D\right)^{2}
\end{gathered}
$$

Proof. To prove (4.22.2), we notice that the coefficients of $B$ and the functions $\xi_{1}^{(0)}$, $\left(\xi_{1}^{(0)}\right)^{-1}$ are elements of $\mathcal{B}_{K}^{(1)}\left(\mathcal{T}_{2} \times D\right)$. Iterating the $u \longmapsto u^{\sigma \phi}$ operation $s$ times, we conclude that $u_{i}^{(0)} \in \mathcal{B}_{K}^{(1)}\left(\mathcal{T}_{2} \times D\left(0,\left(p^{-\frac{1}{s}}\right)^{+}\right)\right)$, for $i=1,2$. The statement follows. To prove (4.22.1) we write $\mathbf{B}$ in the form

$$
\mathbf{B}=\left(\begin{array}{cc}
\lambda^{\mu_{c}} Q_{1} & \lambda^{\mu_{c}} Q_{2} \\
p Q_{3} & p Q_{4}
\end{array}\right) \xi_{2}^{(0)}
$$


where $Q_{1}, Q_{2}, Q_{3}, Q_{4}$ are all bounded by 1 on $\mathcal{T}_{1} \times D$. Hence by (4.21.1) we deduce

$$
\begin{aligned}
& u_{3}^{(0)} u_{3}^{(0)}{ }^{\sigma \phi} Q_{1}+u_{4}^{(0)}{ }^{\sigma \phi} \frac{p}{\lambda^{\mu_{c}}} Q_{3}, \\
& u_{4}^{(0)} u_{3}^{(0)}{ }^{\sigma \phi} Q_{2}+u_{4}^{(0)}{ }^{\sigma \phi} \frac{p}{\lambda^{\mu_{c}}} Q_{4} .
\end{aligned}
$$

Using the maximum modulus theorem we conclude that if $\left(u_{3}^{(0)}, u_{4}^{(0)}\right)$ is bounded by 1 on $\mathcal{T}_{1} \times D\left(0, \rho^{+}\right)$and if

$$
\frac{|p|}{\rho^{\frac{\mu_{c}}{p}}} \leq 1
$$

then $\left(u_{3}^{(0)}, u_{4}^{(0)}\right)$ is bounded by 1 on $\mathcal{T}_{1} \times D\left(0, \rho^{\frac{1}{p}}{ }^{+}\right)$. Since $\mu_{c} \leq p-1$, it follows that $\rho^{\mu_{c}} \geq \rho^{p-1}$ and so (4.22.5) is certainly valid if $\rho \geq|\pi|^{p}\left(<p^{-1}\right)$. This point is not treated properly in $[\mathrm{Dw} 2, \S 2]$.

Q.E.D.

The conclusion is ( $c f$. [ $p$-DE IV, Lemma 2.2]):

Corollary 4.23. The functions $(c-b) F(a, b, c+1 ; \lambda)$ and $c F(a, b, c ; \lambda)$ (resp. $(1-c) F(b-c, a-c, 1-c ; \lambda)$ and $(c-a) \lambda F(1+b-c, 1+a-c, 2-c ; \lambda))$ are elements of $\mathcal{B}_{K}^{(1)}\left(\mathcal{T}_{2} \times D\right)\left(\right.$ resp. $\left.\mathcal{B}_{K}^{(1)}\left(\mathcal{T}_{1} \times D\right)\right)$. As a consequence, for $(a, b, c) \in \mathcal{T}_{2}$ (resp. $\left.(a, b, c) \in \mathcal{T}_{1}\right)$ the elements

$$
F(a, b, c+1 ; \lambda) \quad \text { and } \quad F(a, b, c ; \lambda)
$$

$$
\text { (resp. } F(b-c, a-c, 1-c ; \lambda) \quad \text { and } \quad F(1+b-c, 1+a-c, 2-c ; \lambda))
$$

of $\mathbf{Q}_{p}[[\lambda]]$ are in fact in $1+\lambda \mathbf{Z}_{p}[[\lambda]]$.

Theorem 4.24. For $i=1,2$, the fixed-point $\eta \in \mathcal{E}_{K}^{(1)}\left(\mathcal{T}_{i} \times S_{i}^{H}\right)$ of the contraction $C_{i}$ has the following local expression in $\mathcal{T}_{i} \times D$ :

$$
\eta(a, b, c ; \lambda)= \begin{cases}\frac{u_{4}^{(0)}}{u_{3}^{(0)}}=\frac{c-a}{1-c} \frac{\lambda F(1+b-c, 1+a-c, 2-c ; \lambda)}{F(b-c, a-c, 1-c ; \lambda)} & \text { in } \mathcal{T}_{1} \times D, \\ \frac{u_{1}^{(0)}}{u_{2}^{(0)}}=\frac{c-b}{c} \frac{F(a, b, c+1 ; \lambda)}{F(a, b, c ; \lambda)} & \text { in } \mathcal{T}_{2} \times D .\end{cases}
$$

From the identities $(4.22 .1,2)$ we deduce

$$
\begin{gathered}
\left(u_{3}^{(0)}\right)^{\sigma \phi} \mathbf{B}_{1}+\left(u_{4}^{(0)}\right)^{\sigma \phi} \mathbf{B}_{3}=\xi_{2}^{(0)} \lambda^{\mu_{c}} u_{3}^{(0)} \\
\left(u_{1}^{(0)}\right)^{\sigma \phi} \mathbf{B}_{2}+\left(u_{2}^{(0)}\right)^{\sigma \phi} \mathbf{B}_{4}=\xi_{1}^{(0)} u_{2}^{(0)} \\
\mathbf{B}_{1}+\frac{\left(u_{4}^{(0)}\right)^{\sigma \phi}}{\left(u_{3}^{(0)}\right)^{\sigma \phi}} \mathbf{B}_{3}=\xi_{2}^{(0)} \lambda^{\mu_{c}} \frac{u_{3}^{(0)}}{\left(u_{3}^{(0)}\right)^{\sigma \phi}} \\
\frac{\left(u_{1}^{(0)}\right)^{\sigma \phi}}{\left(u_{2}^{(0)}\right)^{\sigma \phi}} \mathbf{B}_{2}+\mathbf{B}_{4}=\xi_{1}^{(0)} \frac{u_{2}^{(0)}}{\left(u_{2}^{(0)}\right)^{\sigma \phi}}
\end{gathered}
$$


In other words, in $\mathcal{T}_{1} \times D$ we have

$$
\begin{aligned}
& \mathbf{B}_{1}(a, b, c ; \lambda)+\eta^{\sigma \phi}(a, b, c ; \lambda) \mathbf{B}_{3}(a, b, c ; \lambda) \\
& \quad=\xi_{2}^{(0)}(a, b, c) \frac{1-c}{1-c^{\prime}} \frac{F(b-c, a-c, 1-c ; \lambda)}{F\left(b^{\prime}-c^{\prime}, a^{\prime}-c^{\prime}, 1-c^{\prime} ; \lambda^{p}\right)},
\end{aligned}
$$

while in $\mathcal{T}_{2} \times D$ we have

$$
\begin{gathered}
\eta^{\sigma \phi}(a, b, c ; \lambda) \mathbf{B}_{2}(a, b, c ; \lambda)+\mathbf{B}_{4}(a, b, c ; \lambda) \\
\quad=\xi_{1}^{(0)}(a, b, c) \frac{c}{c^{\prime}} \lambda^{\mu_{c}} \frac{F(a, b, c ; \lambda)}{F\left(a^{\prime}, b^{\prime}, c^{\prime} ; \lambda^{p}\right)} .
\end{gathered}
$$

A consequence of the previous formulas is

Theorem 4.28. The function locally defined in $\mathcal{T}_{1} \times D\left(\right.$ resp. $\left.\mathcal{T}_{2} \times D\right)$ by

$$
\begin{gathered}
\mathcal{G}(a, b, c ; \lambda)=\frac{F(b-c, a-c, 1-c ; \lambda)}{F\left(b^{\prime}-c^{\prime}, a^{\prime}-c^{\prime}, 1-c^{\prime} ; \lambda^{p}\right)} \\
\left(\text { resp. } \mathcal{F}(a, b, c ; \lambda)=\frac{F(a, b, c ; \lambda)}{F\left(a^{\prime}, b^{\prime}, c^{\prime} ; \lambda^{p}\right)}\right)
\end{gathered}
$$

extends to an element of $\mathcal{E}_{K}^{(1)}\left(\mathcal{T}_{1} \times S_{1}^{H}\right)\left(\right.$ resp. $\left.\mathcal{E}_{K}^{(1)}\left(\mathcal{T}_{2} \times S_{2}^{H}\right)\right)$. We have in $\mathcal{T}_{1} \times S_{1}^{H}$

$$
\begin{aligned}
\mathcal{G}(a, b, c ; \lambda)=(-1)^{\mu_{c}-\mu_{b}} \frac{\gamma_{p}\left(1+c-a, 1+c^{\prime}-a^{\prime}\right)}{\gamma_{p}\left(c, c^{\prime}\right) \gamma_{p}\left(1-a, 1-a^{\prime}\right)} \lambda^{-\mu_{c}} \\
\cdot\left(\mathbf{B}_{1}(a, b, c ; \lambda)+\eta^{\sigma \phi}(a, b, c ; \lambda) \mathbf{B}_{3}(a, b, c ; \lambda)\right),
\end{aligned}
$$

while in $\mathcal{T}_{2} \times S_{2}^{H}$ we have

$$
\begin{aligned}
\mathcal{F}(a, b, c ; \lambda)= & \frac{\gamma_{p}\left(c, c^{\prime}\right)}{\gamma_{p}\left(b, b^{\prime}\right) \gamma_{p}\left(c-b, c^{\prime}-b^{\prime}\right)} \\
& \cdot\left(\eta^{\sigma \phi}(a, b, c ; \lambda) \mathbf{B}_{2}(a, b, c ; \lambda)+\mathbf{B}_{4}(a, b, c ; \lambda)\right) .
\end{aligned}
$$

\section{Young's FORMUla $(p \geq 7)$}

We plan to put together formulas (2.33) and (4.28.2). This requires a modification of the discussion of the previous section, since as it stands formula (4.28.2) cannot be evaluated at $\lambda=-1$, since $-1 \notin S_{2}^{H}$. We then define

$$
\begin{gathered}
\mathcal{S}_{2}^{\prime}(-1)=\left\{\left(\mu_{a}, \mu_{b}, \mu_{c}\right)=T_{I}(\vec{\mu}) \in \mathcal{S}^{\prime} \mid\right. \\
\left.\vec{\mu} \text { is in case } 2 \text { and }\left|P_{2,2}^{\vec{\mu}}(-1)\right|=1\right\}, \\
\mathcal{T}_{2}(-1)=\left\{(a, b, c) \in \mathcal{T}_{2} \mid\left(\mu_{a^{(j)}}, \mu_{b^{(j)}}, \mu_{c^{(j)}}\right) \in \mathcal{S}_{2}^{\prime}(-1), \forall j=0,1, \ldots\right\},
\end{gathered}
$$

and

$$
S_{2}^{H}(-1)=\left\{\lambda \in S|| P_{2,2}^{\vec{\mu}}(\lambda) \mid=1, \forall \vec{\mu} \in T_{I}^{-1}\left(\mathcal{S}_{2}^{\prime}(-1)\right)\right\} .
$$

The arguments used in section 4 show that 
Corollary 5.2. The function locally defined in $\mathcal{T}_{2}(-1) \times D$ by $\mathcal{F}(a, b, c ; \lambda)=$ $\frac{F(a, b, c ; \lambda)}{F\left(a^{\prime}, b^{\prime}, c^{\prime} ; \lambda^{p}\right)}$ extends to an element of $\mathcal{B}_{K}^{(1)}\left(\mathcal{T}_{2}(-1) \times S_{2}^{H}(-1)\right)$. Formula (4.28.2) holds in $\mathcal{T}_{2}(-1) \times S_{2}^{H}(-1)$, and in particular at $(a, b, c ;-1)$, for any $(a, b, c) \in$ $\mathcal{T}_{2}(-1)$.

We are now supposed to replace $(a, b, c) \in \mathcal{T}_{2}$ in (4.28.2) by $(a, b, 1+a-b) \in \mathcal{T}_{2}$, assuming $\mu_{a^{(j)}}$ even and $\mu_{a^{(j)}} \leq \mu_{b^{(j)}}$ for $j=0,1, \ldots$ We point out that then automatically $(1+a-b)^{(j)}=1+a^{(j)}-b^{(j)}, 2 \mu_{b^{(j)}}-\mu_{a^{(j)}} \leq p-1, \mu_{b^{(j)}}<p-1$, for $j=0,1, \ldots$, and, by $(2.33 .4),(a, b, 1+a-b) \in \mathcal{T}_{2}(-1)$. We now evaluate formula (4.28.2) at $(a, b, 1+a-b ;-1) \in \mathcal{T}_{2}(-1) \times S_{2}^{H}(-1)$, as before. We get

Theorem 5.3. Let $(a, b) \in \mathbf{Z}_{p}^{2}$ be such that $\mu_{a^{(j)}} \leq \mu_{b^{(j)}}<p-1,2 \mu_{b^{(j)}}-\mu_{a^{(j)}} \leq$ $p-1$ and $\mu_{a^{(j)}}$ is even, for $j=0,1, \ldots$ Then $(a, b, 1+a-b) \in \mathcal{T}_{2}(-1) \times S_{2}^{H}(-1)$ and

$$
\mathcal{F}(a, b, 1+a-b ;-1)=(-1)^{\frac{\mu_{a}}{2}} \frac{\gamma_{p}\left(\frac{a}{2}, \frac{a^{\prime}}{2}\right) \gamma_{p}\left(b-\frac{a}{2}, b^{\prime}-\frac{a^{\prime}}{2}\right)}{\gamma_{p}\left(a, a^{\prime}\right) \gamma_{p}\left(b-a, b^{\prime}-a^{\prime}\right)} .
$$

Proof. Direct substitution of formula (2.33.2) in (4.28.2) yields

$$
\begin{gathered}
\mathcal{F}(a, b, 1+a-b ;-1)=(-1)^{\mu_{b}-\frac{\mu_{a}}{2}} \frac{\gamma_{p}\left(1+a-b, 1+a^{\prime}-b^{\prime}\right)}{\gamma_{p}\left(b, b^{\prime}\right) \gamma_{p}\left(1+a-2 b, 1+a^{\prime}-2 b^{\prime}\right)} \\
. \frac{\gamma_{p}\left(\frac{a}{2}, \frac{a^{\prime}}{2}\right) \gamma_{p}\left(b-\frac{a}{2}, b^{\prime}-\frac{a^{\prime}}{2}\right) \gamma_{p}\left(b, b^{\prime}\right)}{\gamma_{p}\left(a, a^{\prime}\right) \gamma_{p}\left(2 b-a, 2 b^{\prime}-a^{\prime}\right)} .
\end{gathered}
$$

The result follows from the reflection formula for $\gamma_{p}(1.20)$.

Q.E.D.

The previous result, restricted to $\mathcal{T}_{2} \cap \mathbf{Q}^{3}$, is essentially theorem 3.1 of [Y2], and generalizes formula $(0.3)$.

\section{REFERENCES}

[AD] A. Adolphson and B. Dwork, The non-regular case-Chapter 9 revisited, preprint Princeton University (1992), 21 pages.

[Ba] F. Baldassarri, $p$-adic formulas and unit root $F$-subcrystals of the hypergeometric system, article in preparation.

[Bo] M. Boyarsky, p-adic gamma function and Dwork cohomology, Trans. Amer. Math. Soc. 257 (1980), 359-369. MR 81c:12029

[Ch] G. Christol, Modules Différentiels et Equations Différentielles p-adiques, Queen's Papere in Pure and Applied Mathematics 66 (1983), 218 pages. MR 87k:11114

[D] J. Diamond, Hypergeometric series with a p-adic variable, Pacific J. Math. 94 (1981), 265-276. MR 82k: 12020

[DR] B. Dwork and Ph. Robba, On ordinary linear p-adic differential equations, Trans. Amer. Math. Soc. 231 (1977), 1-46. MR 56:5562

[Dw1] B. Dwork, On the Boyarsky principle, Amer. J. of Math. 104 (1983), 115-156. MR 85b:11111

[Dw2] B. Dwork, Singular residue classes which are ordinary for $F(a, b, c ; \lambda)$, Groupe d'Étude d'Analyse Ultramétrique, $10^{\text {ème }}$ année, 1982/83, nº 23, 11 pages (Y.Amice, G.Christol, Ph.Robba, eds.). MR 85k:12004

[GHF] B. Dwork, Generalized Hypergeometric Functions, Oxford Mathematical Monographs, Clarendon Press, Oxford, 1990, 188 pages. MR 92h:14006

[GrKo] B. Gross and N. Koblitz, Gauß sums and the p-adic $\Gamma$-function, Annals of Math. 109 (1979), 569-581. MR 80g:12015 
[Ka] N. Katz, Travaux de Dwork, Séminaire Bourbaki $24^{\text {ème }}$ année, 1971/72, no 409, Lecture Notes in Math., 317, Springer-Verlag, Berlin, 1973, pp. 167-220. MR 58:16672

[Ko] N. Koblitz, The hypergeometric function with p-adic parameters, Proceedings of the Queen's (Ontario) Number Theory Conference, 1979, Queen's Papers in Pure and Applied Mathematics 54, 1980, pp. 319-328. MR 83h:12026

[Ku] B. Dwork, On Kummer's twenty-four solutions of the hypergeometric differential equation, Trans. Amer. Math. Soc. 285 (1984), 497-521. MR 86a:12002

[LDE] B. Dwork, Lectures on p-adic Differential Equations, Grundlehren der mathematischen Wissenschaften 253, Springer, 1982, 310 pages. MR 84g:12031

[Mo] Y. Morita, A p-adic analogue of the $\Gamma$-function, J. Fac. Sci. Univ. Tokyo Sect. IA Math. 22 (1975), 255-266. MR 54:12720

$[p-\mathrm{C}] \quad$ B. Dwork, $p$-adic cycles, Publ. Math. IHES 37 (1969), 327-415. MR 45:3415

[p-DE IV] B. Dwork, On p-adic differential equations IV. Generalized hypergeometric functions as p-adic analytic functions in one variable, Ann. Sci. École Norm. Sup. (4) 6 (1973), 295-315. MR 58:27988b

[Po] E.G.C. Poole, Introduction to the Theory of Linear Differential Equations, Dover Publications Inc., New York, 1960, 199 pages. MR 22:2746

[Ro] Ph. Robba, Une introduction naïve aux cohomologies de Dwork, Mem. Soc. Math. France 23 (1986), 61-105. MR 88a:14023

[Sch] A.J. Scholl, A trace formula for F-crystals, Inv. Math. 79 (1985), 31-48. MR 86c:14017

[Sel] F. Baldassarri, p-adic interpolation of Evans sums and deformation of the Selberg integrals: an application of Dwork's theory, Proceedings of the Taniguchi Symposium on Special Differential Equations. Kyoto, September 2-5, 1991, RIMS Publications.

[Y1] P.Th. Young, Apéry numbers, Jacobi sums, and special values of generalized p-adic hypergeometric functions, J. of Number Theory 41 (1992), 231-255. MR 93j:11079

[Y2] P.Th. Young, On Jacobi sums, multinomial coefficients, and p-adic hypergeometric functions, J. of Number Theory 52 (1995), 125-144. CMP 95:12

Dipartimento di Matematica, Università di Padova, Via Belzoni 7, I-35131, Padova, ITALY

E-mail address: baldassarri@pdmat1.math.unipd.it 